\title{
QTL Analysis of Multiple Behavioral Measures of Anxiety in Mice
}

\author{
Norman D. Henderson, ${ }^{1,4}$ Maria Grazia Turri, ${ }^{2}$ John C. DeFries, ${ }^{3}$ and Jonathan Flint ${ }^{2}$
}

Received 2 Dec. 2002-Final 31 July 2003

In a test battery consisting of an open-field arena, a light-dark box, a mirror-chamber box, an elevated plus maze, and an elevated square maze, 1,671 mice were tested, generating over 100 putative measures of anxiety in rodents. Quantitative trait loci (QTL) analysis was carried out on all measures, plus composite measures and phenotypic factor scores. Significant LOD scores were found for QTL on 17 chromosomes, with large and consistent QTL behavioral effects on chromosomes 1, 4, 7, 8, 14, 15, 18, and X. QTL on chromosomes 4 and 8 largely influence locomotor activity in both home cages and novel environments, whereas QTL on chromosomes 1, 15, and 18 influence anxiety-related behaviors. Five genetically separable, cross-test dimensions of anxiety could be identified: (i) the suppression of locomotor activity in low to moderately anxiogenic regions of the tests; (ii) a shift toward proportionally less time and activity spent in high-anxiogenic test areas; (iii) the suppression of rearing behavior; (iv) increased latency to enter novel areas; (v) increased autonomic responses, as assessed by defecation and urination. Patterns of QTL influence on cross-test composite scores were distinctive. For example, the QTL on chromosome 1 strongly influenced safe-area locomotor activity $(\mathrm{LOD}=35)$ and autonomic responses $(\mathrm{LOD}=16)$, whereas the QTL on chromosome 15 influenced the proportion of activity in high-anxiogenic areas $(\mathrm{LOD}=16)$, latency to enter novel areas (LOD $=36)$ and rearing behavior (LOD = 57). Phenotypic factor analysis identified factors heavily loaded on single tests, rather than cross-test factors. The use of factor analysis or within-test principal components for data reduction before genetic analysis was less satisfactory than using genetic dissection methods on the original measures and logically derived composites.

KEY WORDS: Open-field; light-dark box; elevated plus maze; elevated square maze; mirror chamber; anxiety; activity; emotionality; autonomic responses; anxiogenic environments; rearing; risk taking; home cage; quantitative trait loci (QTL); genetic dissection; chromosome; LOD scores; F2 mice; factor analysis.

\section{INTRODUCTION}

During the past decade we have been using molecular mapping strategies to investigate the genetic architecture responsible for individual behavioral differences in the DeFries strains of mice (Flint et al., 1995; Turri et al., 2001a, b). The DeFries strains are derived from a cross of two inbred strains (BALB/cJ and C57/BL6)

${ }^{1}$ Oberlin College, Oberlin, Ohio.

${ }^{2}$ Wellcome Trust Centre for Human Genetics, Oxford, United Kingdom.

${ }^{3}$ Institute for Behavioral Genetics, University of Colorado, Boulder, Colorado.

${ }^{4}$ To whom correspondence should be addressed at Department of Psychology, Oberlin College, Oberlin, Ohio 44074. Tel: 440-7757695; Fax: 440-775-8356. e-mail: nhenders@oberlin.edu that were selected for differences in open-field activity, a test that is believed to model susceptibility to anxiety in rodents. Thirty generations of artificial selection in the open-field arena resulted in large activity differences among the lines, which persisted after 18 generations of random mating and 35+ generations of inbreeding, that produced the six DeFries strains: two high (H1 and H2), two low (L1 and L2), and two control lines ( $\mathrm{C} 1$ and $\mathrm{C} 2)$, that were not subject to selection (DeFries and Hegman, 1970; DeFries et al., 1978).

We have previously reported genetic mapping experiments using $\mathrm{F} 2$ intercrosses between both the $\mathrm{H} 1$ and L1 strains and the $\mathrm{H} 2$ and L2 strains, using several phenotypes to determine whether a common set of genetic loci influence behavior in tests that are believed 
to measure variation in anxiety in mice (Turri et al., 2001b). Behavioral tests included the open-field arena, the elevated plus maze, (Pellow et al., 1985), the square-maze, (Shepherd et al., 1994) the light-dark box (Costall et al., 1989), and the mirror chamber box (Toubas et al., 1990). We found that genetic loci on chromosomes 1, 4, 15, and 18 influenced at least one measure obtained in all five tests, validating the assumption that there is a psychological construct, sometimes termed emotionality, influencing anxiety-related behavior (Turri et al., 2001b). In addition, the large size of our study, including over 1600 mice and a replicate of a previous intercross between L1 and H1 strains (Flint et al., 1995), provided a powerful test of the robustness of quantitative trait loci (QTL) mapping strategies (Turri et al., 2001a, b).

The large data set we have acquired allows us to address a number of other questions. In this report we explore the consequences of the combination and choice of phenotypes for genetic mapping. In accompanying papers we investigate multivariate approaches to genetic mapping and the importance of epistatic interactions.

A critical feature in any QTL analysis is the selection, and definition, of phenotypes for mapping. This is not only because we must be clear about what we are mapping, as Nadeau and Frankel point out (2000). There are two additional reasons to investigate the choice of phenotypes: first, it can influence the power to detect QTL and determine mapping resolution, as the genetic architecture of phenotypes may vary. For instance, although two traits may have similar heritabilities, one may be influenced by many loci, each of small effect, whereas the other is influenced by a single locus of relatively large effect. The latter offers an easier target for further molecular dissection. Second, the inclusion of many correlated phenotypes for genetic mapping can be used to carry out what has been termed "physiological profiling" (Stoll et al., 2001), to interpret the phenotypes in the light of genetic analysis. Genetic interpretation is particularly useful for behavioral analysis where, often, little is known of the physiology.

One way of utilizing multiple phenotypes is to extract common factors, using factor analysis. However, the value of this approach in genetic mapping of fearful behavior is not clear. It is generally assumed that the various tests of anxiety in rodents, such as the elevated plus maze, open-field arena, and conditioned fear paradigms, measure at least one common underlying trait. Consistent with this hypothesis, Trullas and Skolnick (1993) report that performance in the elevated maze predicts behavior in other animal models of anxiety. In a study comparing inbred mouse strains, they found significant negative correlations between the time spent in the open arms of the elevated maze and amplitude of an acoustic startle or latency to eat in a hyponeophagia test. However, a difficulty with factor analysis of test battery variables is that the procedure does not always identify a predicted common element. More often the factors reported largely correspond to clusters of measures taken within each specific test in the battery (e.g., Aguilar et al., 2002; Fernandes et al., 1999; Flaherty, 1998; Royce et al., 1973).

In this paper, we report genetic mapping of all phenotypic data collected during the study, providing a complete picture of genetic effects on over 100 phenotypes. We extend the process of genetic validation to multivariate factors created by combining common behaviors observed across test environments and compare this procedure to using traditional factor analysis of phenotypic correlations for purposes of data reduction before genetic analysis. In addition to using factor analysis and principal components, we investigate combining phenotypes believed to measure the same latent trait, rather than relying on factor analysis to group the phenotypes.

\section{METHODS}

\section{Subjects}

Mice used in this study consisted of two F2 intercrosses of the DeFries strains of mice (DeFries et al., 1978). These strains are inbred derivatives of lines subjected to 30 generations of bi-directional selection in the open field. Selection was carried out in two separate experiments so that there are four lines, termed $\mathrm{H} 1$ and $\mathrm{H} 2$ (for high activity) and L1 and L2 (for low activity). Complete behavioral and genotyping data were obtained from $815 \mathrm{~F} 2$ mice derived from the $\mathrm{H} 1 \times \mathrm{L} 1$ strains and 821 mice derived from the H2 $\times$ L2 strains (Turri et al., 200la, b). The number of male and female mice was approximately equal in each replicate set. All mice were bred in the specific pathogen-free colony of the Institute for Behavioral Genetics, University of Colorado, Boulder, Colorado.

\section{Apparatus and Test Procedures}

\section{General Procedures for All Behavioral Tests}

The Principles of Laboratory Animal Care (NIH publication No. 86-23, revised 1985) were followed, and all research protocols were approved by the 
University of Colorado, Boulder, Animal Care and Use Committee. At 27 to 38 days of age, mice were moved from the main colony room to the QTL testing colony area, which was a partitioned section of the animal testing facility designed for this project. Mice were housed with like-sex littermates (two or three per cage). Between day 61 and day 69, littermates were weighed, tail marked (Marks-a-Lot permanent marker), and provided with a fresh group cage. The following day, testing of mice began in the anxiety battery. All behavioral testing was done during the first $5 \mathrm{~h}$ of the dark cycle, excluding the first $30 \mathrm{~min}$ of the cycle. Mice were moved from colony cage racks to the adjacent testing area $30 \mathrm{~min}$ before testing commenced. Each mouse received one test every 24 to $48 \mathrm{~h}$ in the fixed order: open-field arena (OF1), light-dark box (LD1), elevated plus maze (EP), mirror-chamber box (MC), elevated square maze (ES), open-field arena retest (OF2), and light-dark box retest (LD2). All mice completed the seven-test battery in 7 to 12 days.

Test duration was $5 \mathrm{~min}$ in each apparatus. In every apparatus, each beam break was time-stamped, allowing software to do a complete analysis of time and activity patterns in all areas of the test field. A high level of photo emitter/detector coverage in each apparatus provided redundant systems to maximize reliability of monitoring. In addition, the software provided photo detector reliability checks and logic checks of detector sequences, which described the paths traversed by the test animal. Mice were removed promptly at the end of the test session and the number of fecal boli counted and urination scored, using a 0 to 3 scale (none to throughout apparatus). The test apparatus was then cleaned with a $70 \%$ EtOH solution, wiped dry, and allowed to air dry before next use.

Care was taken to distribute testing of F2 genotypes from the four $\mathrm{F} 1$ parental combinations $(\mathrm{HL} \times$ $\mathrm{HL}, \mathrm{HL} \times \mathrm{LH}, \mathrm{LH} \times \mathrm{HL}, \mathrm{LH} \times \mathrm{LH}$ ) evenly throughout the 12-month testing period. Statistical analysis verified that this was the case-the relationship between reciprocal-cross genotype and birth date approached 0 $($ eta $=.03)$, and the phi coefficient based on the distribution of parental genotypes across five test periods was .13. Replicates were also evenly distributed across test periods (eta $=.09$, phi $=.10)$.

\section{Open-Field Arena}

The open-field arena is a 61-cm-white plastic lined square box, $38 \mathrm{~cm}$ deep. The apparatus is covered and a compact fluorescent bulb in the center of the ceiling provided an illuminance of approximately $600 \mathrm{~lx}$ on the floor below. The field is divided into an $8 \times 8$ grid of 76-mm squares using infrared emitters and photodetectors to monitor horizontal movement. In addition, four emitters at each corner are placed $6 \mathrm{~cm}$ from the floor to detect rearing on and near the walls of the open field. Animals are placed in a Plexiglas start cylinder in one corner of the apparatus; the cylinder is removed, the lid closed, and activity monitored for $5 \mathrm{~min}$. The test apparatus differs from the open field originally used for selection of the progenitor lines of the DeFries strains. Although the two fields used a similar illumination level, the original field was larger $\left(91 \mathrm{~cm}^{2}\right)$ and not sound attenuated. The testing duration used during selection was $3 \mathrm{~min}$, rather than the $5 \mathrm{~min}$ used in the present study.

\section{Light-Dark Box}

The light-dark box $(27 \times 45 \mathrm{~cm})$ is divided into two chambers. The black-walled, dark chamber is $27 \times$ $15 \mathrm{~cm}$ and has an 8-cm-wide and 9-cm-high opening in the middle of the wall adjoining the $27-\times 30-\mathrm{cm}$ whitewalled lighted chamber. A small, shaded fluorescent bulb, positioned at the top of the divider wall in the light compartment provides $20 \mathrm{~lx}$ illuminance to the light side of the box. Infrared emitter/detectors record horizontal activity in both light and dark compartments. Animals were placed in the dark chamber, a red plastic lid closed over the apparatus, and recording begun. Latency to emerge from the dark compartment, time and activity in each compartment, and light-dark compartment transitions were recorded.

\section{Elevated Plus Maze}

The elevated plus maze consists of four runways $(5 \mathrm{~cm} \times 30 \mathrm{~cm})$ arranged in a cross and elevated $37.5 \mathrm{~cm}$ above the ground. The runway floors were constructed of textured translucent plastic. Two of the arms are enclosed by $21-\mathrm{cm}$ clear acrylic plastic walls, and two arms are open, except for a slight raised $(0.25 \mathrm{~cm})$ edge, which essentially eliminated the problem of mice falling from the open runways. A low-output compact fluorescent bulb, located $1 \mathrm{~m}$ above the center of the maze, provided approximately $20 \mathrm{~lx}$ illuminance on all runways of the maze. The clear walls allow equal illumination levels on both open and closed runways, thus avoiding a confounding of light aversion and openclosed runway preferences. Infrared emitter/detector pairs located around the perimeter of the apparatus detect movement. Beams are positioned to detect both horizontal activity and vertical rearing in each runway and scanning over the edges and ends of open runways. Animals are placed in a clear rectangular bottomless 
start box in the center of the maze. The start box is lifted and testing begun. Locomotor activity, rearing, scanning, and time spent in each arm are recorded, as well as transitions into different arms.

\section{Mirror Chamber Test}

The mirror chamber, designed to detect anxiolytic agents, is based on the principle that many species show approach-avoidance conflict behavior when faced with a mirror image (Toubas et al., 1990). The outer box containing the chamber is constructed of black plastic, $40 \mathrm{~cm} \times 40 \mathrm{~cm} \times 30.5 \mathrm{~cm}$ high. Located within this box is a black $30.5-\mathrm{cm}$ cube, open on one end. The three inner walls, ceiling, and floor of the cube are mirrored. The illuminance within the mirrored chamber was approximately $10 \mathrm{~lx}$. The space between the inner cube and outer box provides the animal with a $4.6-\mathrm{cm}$ darkwalled dim (1-2 lx) alley surrounding the cube. The mouse is placed in the narrow alley at the farthest point from the opening to mirrored chamber. Infrared emitterdetectors monitored alley-to-alley transitions, rearing and latency to enter the mirrored chamber.

\section{Elevated Square Maze}

The elevated square maze differs from the elevated plus maze in providing a continuous circuit for the animal to traverse, with alternating enclosed and open regions. Floors of the square maze were constructed of smooth black plastic, to contrast from those of the plus maze. The maze is a $35-\mathrm{cm}$ square, with $5-\mathrm{cm}$-wide runways, two of which, on opposing sides, are enclosed by $20-\mathrm{cm}$ clear plastic walls. The open runways contained $.25 \mathrm{~cm}$ edges, similar to those on the plus maze. Illumination level and infrared activity monitoring methods were the same as those described for the elevated plus maze. Animals are placed in a bottomless start box in a corner of one closed arm. The start box is lifted and mice tested for five minutes. All activity and time spent in each arm, as well as transitions into different arms, is recorded.

\section{Home Cage Activity Monitoring}

The day following the completion of the test battery, each mouse was given a 3-min tail-hang test. Two days later, littermates were weighed and separated into individual cages identical to those used for group housing. After 24 to $48 \mathrm{~h}$ of individual housing, each animal's home cage was placed in a rack containing monitoring equipment designed to unobtrusively measure in-cage activity. Mice were 72 to 80 days of age at time of home cage monitoring. Activity was measured during the first $2 \mathrm{~h}$ of the dark cycle on 2 consecutive days. The location of the home cage within the testing rack was changed between day 1 and day 2, to reduce location effects.

Two different activity-monitoring systems were used simultaneously. The first consisted of two infrared photo emitter/detector pairs, located outside the clear plastic home cage that divided the cage into three equal areas. This photocell system recorded activity in a manner similar to that used in the test apparatus, recording gross locomotor activity on the floor of the home cage as the animal broke the infrared beams. The beam-break activity score consisted of the square root of number of beam breaks for each detector summed across the 2 test days. Spearman-Brown corrected test-retest reliability of the measure was .70. The second home cage activity monitoring system employed a motion detector located above the top of the home cage. This system converted all motion sensed within the home cage into arbitrary power units, based on the magnitude of activity detected. For example, rapid gross motor activity, such as climbing on wire cage lids, and cage floor locomotion generated more score units per second than grooming or nest activity. Unlike the beam-break system, which only monitored across cage floor locomotion, mice showing high levels of activity restricted to one area of the home cage could obtain relatively high activity scores with the motion detector. Spearman-Brown corrected test-retest reliability of activity scores based on the motion detector was .79. The correlation between the beam-break and motion detector activity scores was .52 across the 2 test days.

\section{Genotyping and Statistical Analyses}

No new genotyping was conducted for this study. The genetic data are the same as those reported in Turri et al. (2001a, b), involving 1636 of the 1671 mice that were phenotyped in the behavioral battery. Linkage analyses were performed using the interval mapping implemented in QTL-MAPMAKER (Lincoln et al., 1992) as previously described.

\section{RESULTS}

\section{Factor Analysis of Phenotypic Correlations Among Behavioral Measures}

Table I displays the oblique rotated factor loadings on 11 factors obtained from a maximum likelihood factor analysis (SPSS 11.0) of behavioral variables obtained from all tests. Complete data were available for $96.6 \%$ of the total sample of 1671 mice from the two replications. Mean substitution was used for the 
Table I. Oblique Rotated Factor Loadings for Behavioral Phenotypes Obtained from Seven Test Sessions ${ }^{a}$

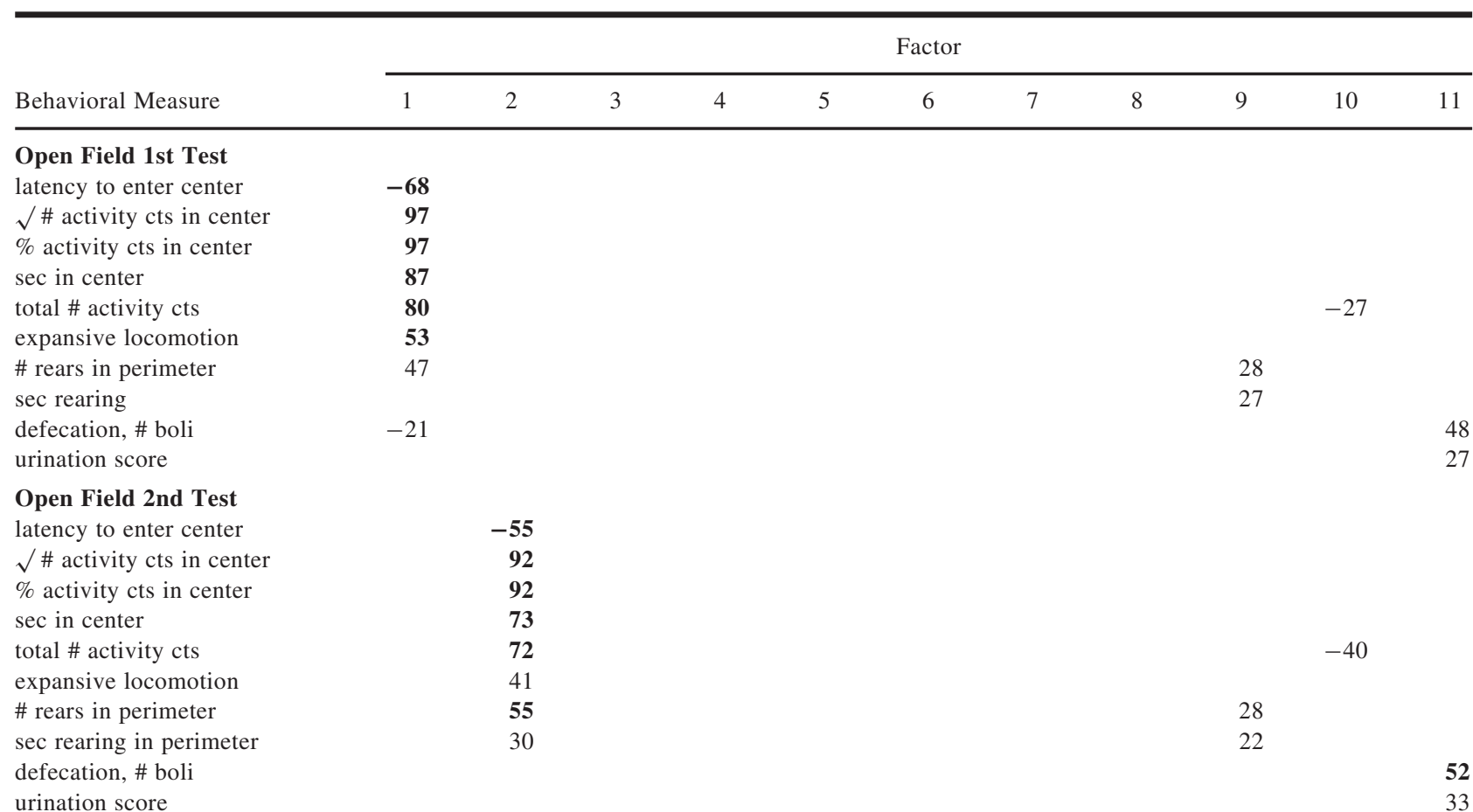

Light-Dark Box, 1st Test

sec to enter light side

dark-light transitions activity cts on light side

sec on light side

$\%$ light activity cts

activity cts on dark side

$\sqrt{ }$ defecation, \# boli

urination score

Light-Dark Box, 2nd Test

sec to enter light side

dark-light transitions

activity cts on light side

sec on light side

$\%$ light activity cts

activity cts on dark side

$\sqrt{ }$ defecation, \# boli

urination score

\section{Mirror Box}

latency to enter mirror room

\# alleys entered

$\sqrt{ } \#$ rears

sec rearing

$\sqrt{ }$ defecation, \# boli

urination score

\section{Elevated Plus Maze}

\# closed arm activity cts

$\sqrt{ } \#$ open arm activity cts

$\%$ activity cts in open arms

sec in open arms

sec in center square

$\sqrt{ } \#$ cts in ends of open arms

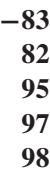

$-51$

48

53

$-59$

$71-32$

$89-23$

$84-20$

94

$-61$

56

$-31$

$45-25$

97

96

$-23$

41

52 
Table I. Continued

\begin{tabular}{|c|c|c|c|c|c|c|c|c|c|c|c|}
\hline \multirow[b]{2}{*}{ Behavioral Measure } & \multicolumn{11}{|c|}{ Factor } \\
\hline & 1 & 2 & 3 & 4 & 5 & 6 & 7 & 8 & 9 & 10 & 11 \\
\hline sec in ends of open arms & & & & 78 & & & & -27 & & & \\
\hline \# entries into open arms & & & 71 & 25 & & & & & & & \\
\hline \# entries to ends of open arms & & & 44 & 54 & & & & & & & \\
\hline$\sqrt{ } \#$ scans over open arms & & & 45 & 44 & & & & & & & \\
\hline \# scans over open arm ends & & & & 91 & & & & & & & \\
\hline log sec scanning over ends & & & & 87 & & & & & & & \\
\hline latency to enter open arm & & & -54 & & & & & & & & \\
\hline $\log$ latency to enter 2 nd arm & & & -45 & & & & & & & & \\
\hline \# rears in closed arms & & & & & & & & 36 & 35 & & \\
\hline sec rearing in closed arms & & & & & & & & 30 & 34 & & \\
\hline \# rears in open $\operatorname{arms}(\log X+1)$ & & & & & & & & & & & \\
\hline defecation, \# boli & & & & & & & & & & & 49 \\
\hline urination score & & & & & & & & & & & 38 \\
\hline Elevated Square Maze & & & & & & & & & & & \\
\hline \# closed arm activity cts & & & & & 45 & & & & & -41 & \\
\hline$\sqrt{ } \#$ open arm activity cts & & & & & 97 & & & & & & \\
\hline$\%$ open arm cts & & & & & 83 & & & & & & \\
\hline sec in corners & & & & & -51 & & & & & & \\
\hline$\sqrt{ }$ scans over open arms & & & & & 79 & & & & & & \\
\hline$\sqrt{ }$ sec scanning, open arms & & & & & 55 & & & & & & \\
\hline latency to enter an open arm & & & & & -40 & & & & & & \\
\hline \# rears in closed arms & & & & & 34 & & & & & & \\
\hline sec rearing in closed arms & & & & & & & & & & & \\
\hline \# rears in open arms $(\log X+1)$ & & & & & 41 & & & & & & \\
\hline defecation, \# boli & & & & & & & & & & & 54 \\
\hline urination score & & & & & & & & & & & 44 \\
\hline Home Cage Activity & & & & & & & & & & & \\
\hline Photo emitter/detector cts & & & & & & & & & & -24 & \\
\hline Motion detector score & & & & & & & & & & & 24 \\
\hline
\end{tabular}

Note: Loadings are based on Maximum Likelihood Factor Analysis with Oblimin Rotation. Decimal points and factor loadings below .2 are omitted. Factor correlations: $r_{12}=.45 ; r_{34}=.43 ; r_{45}=.35 ; r_{16}=.34 ; r_{67}=.33$. All other factor correlations <.30.

occasional missing scores in the remaining mice. Variables used in the QTL analysis that represent linear combinations of other measures taken in the same test apparatus are omitted from this factor analysis. All loadings of 0.2 or larger are shown, with loadings greater than 0.5 in boldface. The 11 factors, which were extracted based on Cattell's scree test (Cattell, 1966), accounted for $62 \%$ of the total variance of the 77 variables. The four largest eigenvalues were 15.3, 6.4, 4.3, and 4.2, and the smallest was 1.6. Six additional factors with eigenvalues between 1.0 and 1.3 are not included in Table I because these factors accounted for trivial percentages $(1.3 \%-1.7 \%)$ of the total variance and added nothing of substance to interpretation of the analysis.

The results illustrate the phenomenon of "instrument factors" usually associated with the factor analysis of measures from multiple test apparatus (e.g.,
Aguilar et al., 2002; Fernandes et al., 1999; Flaherty, 1998; Royce et al., 1973). We suggest that "test session factors" is a more appropriate description of the phenomenon, because repeated test sessions on the same apparatus also produce separate factors. Activity measures from the two open-field arena test sessions load on separate factors ( 1 and 2), as do the two light-dark box test sessions (factors 6 and 7). In both cases, there is a modest correlation between the Session 1 and Session 2 factors $\left(r_{F 1 F 2}=.45, r_{F 6 F 7}=.33\right)$. Activity on the elevated plus maze is represented in correlated factors 3 and 4 $\left(r_{F 3 F 4}=.45\right)$, which largely reflect open-arm activity, and factor 8 , which is related to closed-arm activity. Activity in the elevated square maze makes up factor 5, with no distinctions between open and closed runways.

The remaining three factors in Table I transcend individual tests. Factor 9 reflects rearing behavior across all test apparatus. Factor 10 reflects general 
activity in nonthreatening sections of the various test apparatus and includes a modest loading on home cage locomotor activity assessed with photodetectors. Factor 11 is an autonomic (defecation/urination) factor, reflected in all tests. Factor 11 also has a low loading on scores obtained by the home cage motion detector system, which records a composite of gross motor activity, such as climbing and locomotion, and smaller movements, such as rearing, grooming, and activity within nests.

Despite the large sample size and the clarity of the factors identified, the factor pattern obtained from phenotypic correlations does not provide much insight for understanding the anxiety construct we are attempting to assess with our behavioral measures. Alternative factor extraction and rotation procedures and varying the number of factors extracted led to the same conclusion.

We also carried out single apparatus factor analyses for those test apparatus in which many variables are available. For example, the elevated plus maze produced six factors with eigenvalues greater than 1.0two correlated (.60) open arm (time, activity, entry, scanning) factors, a closed arm factor, and three factors each largely defined by a single correlation coefficient (open arm, rears, closed arm rears, latency to open arm, and latency to second arm). The defecationurination correlation was too low to define a possible fourth single-coefficient factor. Thus, even with a large number of measures available, single apparatus factor analyses tend to produce a combination of underdetermined factors plus a few factors consisting largely of experimentally contingent or linked (e.g., time and activity; open runway and ends of open runway) measures, assessed in a single set of environmental conditions. These analyses are no more informative, with respect to defining the construct of anxiety in rodents, than the phenotypic factor analysis of the full test battery.

\section{QTL Influencing Behavioral Phenotypes Assessed in the Test Battery}

To determine whether there are QTL that influence behavior in our full set of putative measures of anxiety, we obtained genotypic marker data from 1,636 animals from the two replicate intercrosses $(\mathrm{N} 1=815$, $\mathrm{N} 2=821)$ and conducted genetic linkage analyses as previously described (Turri et al., 2001a, b). Since results from the two crosses were consistent, we combined data from the two replicates to maximize power and mapping resolution.
Chromosomes 9, 10, and 19 produced no QTL with LOD scores greater than 2.8 and are thus excluded from this report. Each of the remaining 17 chromosomes had at least one LOD score exceeding 4.3. The appendix contains tables, organized by test apparatus, that list all LOD scores of 2.8 or larger for these 17 chromosomes. For a single phenotype mapped in an F2 intercross, Lander and Kruglyak (1995) argued that LOD scores between 2.8 and 4.3 provide suggestive evidence for the presence of a single QTL and LOD scores greater than 4.3 represent significant evidence. The large numbers of phenotypes we have mapped means that these significance levels cannot be strictly applied; our previous analyses indicated that a LOD score greater than 3.4 is sufficient to keep experiment-wise error rates at $p<0.01$ (Turri et al., 2001a, b). In the remainder of this paper, we highlight results for eight chromosomes that account for the largest proportion of genetic variance for the behavioral variables examined in this experiment.

Tables II through VI list all LOD scores of 2.8 or greater and direction of effects of QTL for chromosomes $1,4,7,8,14,15,18$, and $\mathrm{X}$ for measures obtained from individual test sessions. The five tables are organized by behavioral category, cutting across test apparatus: (i) general locomotor activity and activity in low anxiogenic areas of a test apparatus; (ii) activity in threatening areas of a test apparatus; (iii) latency to enter a novel, and possibly threatening, area in the apparatus; (iv) autonomic behavior, as evidenced by defecation and urination; and (v) rearing behavior. These five broad behavioral traits were found to provide a parsimonious description of QTL effects observed across chromosomes and tests. Although we had both activity-in-area and time-in-area measures for specific regions of each test apparatus, only activity count scores are included in Tables II through V. For these four behavioral categories, time-in-area measures were consistent with activity-in-area measures but generally tended to have lower LOD scores than their counterpart activity-in-area scores. Time-in-area scores and other measures omitted from Tables II through V can be found in the appendix tables.

Table VII lists all LOD scores for multitest composite measures based on two or more scores within Tables II through VI. Composite measures were derived by summing $\mathrm{z}$ scores of comparable measures across tests or were based on composites of standardized regression residuals, as noted in Table VII. Because the number of animals used is large and nearly equal for each phenotype, the relationship between LOD score and approximate percentage of phenotypic 
Table II. LOD Scores and Direction of Effect of QTLs Influencing General Locomotor Activity

\begin{tabular}{|c|c|c|c|c|c|c|c|c|}
\hline \multirow[b]{2}{*}{$\begin{array}{l}\text { Location cM (15th-85th percentile })^{a} \\
95 \% \text { CI of highest LOD trait }{ }^{b}\end{array}$} & \multicolumn{8}{|c|}{ Chromosome } \\
\hline & $\begin{array}{c}1 \\
74-80 \\
74-78\end{array}$ & $\begin{array}{c}4 \\
34-47 \\
31-41\end{array}$ & $\begin{array}{c}7 \\
29-59 \\
49-55\end{array}$ & $\begin{array}{c}8 \\
52-66 \\
56-68\end{array}$ & $\begin{array}{l}14 \\
-{ }^{c} \\
-c\end{array}$ & $\begin{array}{c}15 \\
20-22 \\
19-25\end{array}$ & $\begin{array}{c}18 \\
23-32 \\
20-28\end{array}$ & $\begin{array}{c}X \\
10-50 \\
44-56\end{array}$ \\
\hline $\begin{array}{l}\text { OF1: total \# activity cts } \\
\text { OF1: } \sqrt{ } \text { perimeter activity cts/min } \\
\text { OF2: total \# activity cts } \\
\text { OF2: } \sqrt{ } \text { perimeter activity cts/min } \\
\text { LD1: total \# activity cts } \\
\text { LD1: activity cts on dark side } \\
\text { LD1: activity cts/min on dark side } \\
\text { LD2: total \# activity cts } \\
\text { LD2: activity cts on dark side } \\
\text { LD2: activity cts/min on dark side } \\
\text { PL: \# closed arm activity cts } \\
\text { PL: closed arm activity cts/min } \\
\text { PL: total open + closed activity cts } \\
\text { PL: total open + closed arm entries } \\
\text { SQ: \# closed arm activity cts } \\
\text { SQ: total open + closed activity cts } \\
\text { SQ: total open + closed arm entries } \\
\text { MR: \# alleys entered }\end{array}$ & $\begin{array}{c}27.4-{ }^{d} \\
16.5- \\
24.3- \\
16.3- \\
30.9- \\
7.7- \\
17.8- \\
23.0- \\
11.1- \\
12.6- \\
6.7- \\
15.7- \\
17.5- \\
19.7- \\
21.3- \\
22.5- \\
11.6-\end{array}$ & $\begin{array}{r}3.5- \\
4.6- \\
10.2- \\
8.3- \\
6.3- \\
6.4- \\
6.1- \\
12.2- \\
7.0- \\
7.9- \\
6.5- \\
3.5- \\
7.5- \\
6.3- \\
3.2- \\
4.4- \\
3.5-\end{array}$ & $\begin{array}{r} \\
8.3+ \\
8.1+ \\
6.9+ \\
15.8+ \\
16.5+ \\
11.8+ \\
11.5+ \\
9.7+\end{array}$ & $\begin{array}{l}4.4+ \\
6.2+ \\
8.3+ \\
6.9+ \\
6.1+ \\
5.1+ \\
4.0+ \\
2.7+\end{array}$ & $2.8+$ & $\begin{array}{r}12.5- \\
13.7- \\
5.4- \\
4.0- \\
14.5- \\
5.1- \\
14.4-\end{array}$ & $\begin{array}{r}5.4- \\
5.3- \\
9.2- \\
7.8- \\
5.4- \\
\\
4.6- \\
5.7- \\
2.9- \\
4.6- \\
6.4- \\
9.8- \\
11.8- \\
12.0- \\
10.4- \\
10.4- \\
6.9- \\
4.8-\end{array}$ & $\begin{array}{l}5.3+ \\
4.5+ \\
5.1+ \\
4.4+ \\
3.2+ \\
3.9+ \\
4.0+ \\
6.9+ \\
7.0+ \\
7.7+\end{array}$ \\
\hline $\begin{array}{l}\text { Home cage activity measures } \\
\text { Emitter/detector beam breaks } \\
\text { Motion detector activity score }\end{array}$ & $3.5+$ & $\begin{array}{l}6.8- \\
4.2-\end{array}$ & $4.0+$ & $4.7+$ & $3.9-$ & & $3.0+$ & $4.4+$ \\
\hline
\end{tabular}

${ }^{a} 15$ th and 85 th percentiles of the distribution of peak locations for LOD scores listed for each trait, excluding home cage activity.

${ }^{b}$ CI based on trait with highest LOD score within the 15 th-85th percentile interval.

${ }^{c}$ Too few significant LOD scores for a determination.

${ }^{d}$ Percentage of phenotypic variance accounted for is approximately $1 / 3$ of the LOD score for all QTL.

Footnotes a through d apply to Tables II-VI.

variance explained is a constant $(\%$ variance $=\mathrm{LOD} / 3)$ in Tables II through VII and in the appendix tables. One can also use Darvasi and Soller's (1997) Equation $4,(\mathrm{CI}=530 / \mathrm{Nv})$ to approximate the $95 \%$ confidence interval for our QTL location estimates. The equation tends to overestimate the expected $\mathrm{CI}$ for values less than $10 \mathrm{cM}$ and underestimate the expected $\mathrm{CI}$ for values greater than $10 \mathrm{cM}$. Their Equation 4 simplifies to $\mathrm{CI} \approx 1 / \mathrm{LOD}$ for our combined sample.

The direction of effect indicated for each QTL is that produced by the allele originating from the low active parent strain, L1 or L2. A negative sign following a LOD score indicates that the L-parent allele decreases expression of that behavior and a positive sign indicates increased trait expression from the L-parent allele. In nearly all cases, the directions of individual trait expressions were consistent with an interpretation of a QTL influence on either anxiety or general activity. Most of the QTL effects in Tables II through VII are self-evident; thus we only briefly highlight some of the results here.

\section{Total Locomotor Activity and Activity in Low Anxiogenic Areas of a Test Apparatus}

Table II shows LOD scores of both total locomotor activity measures, assessed throughout all areas of each test apparatus, and activity assessed only in the more protected, and presumably less anxiogenic, areas of each apparatus. With the exception of the QTL on chromosome 7 (Ch7 QTL), which we address below, the direction of effect was completely consistent for all QTL for all chromosomes in Table II. In contrast to this across-test consistency of QTL effects on locomotor activity, we note that the phenotypic correlations of total activity measures in different tests were typically below .30, which was modest enough to produce separate phenotypic factors for each apparatus (Table I).

The exception to this direction of effect consistency, found on Ch7 QTL, involves the suppression of activity in the brightly illuminated open field, which contrasts to increased activity in other tests listed in Table II, all of which used considerably lower illumination levels. The Ch7 QTL is likely to involve, or be 
Table III. LOD Scores and Direction of Effect of QTLs Influencing Activity in Threatening Areas of Test Apparatus

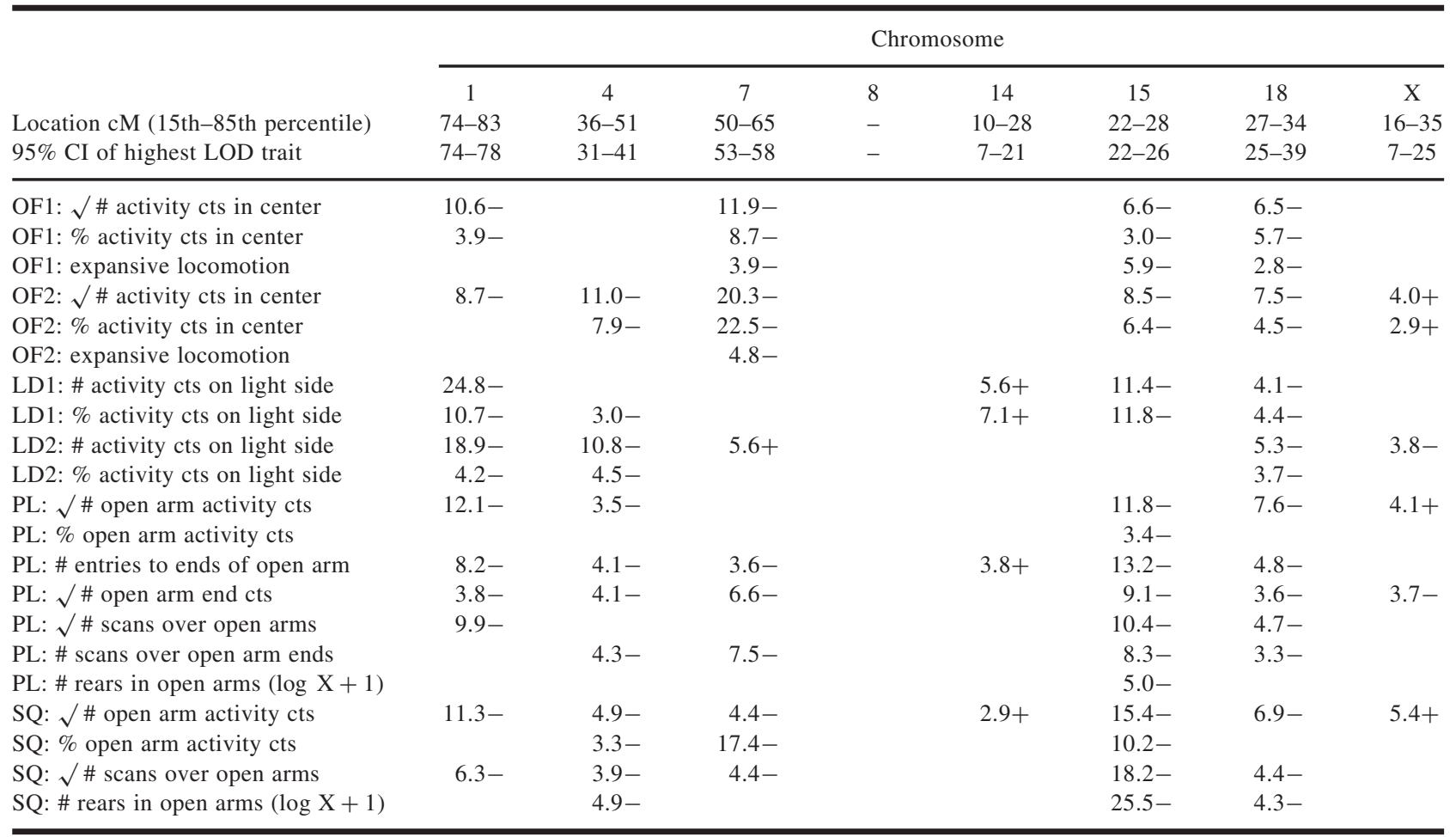

closely linked to the albino (tyr) locus, located $52 \mathrm{cM}$ from the beginning of chromosome 7 , based on our markers. This QTL is a nearly perfect predictor of recorded albino/pigmented coat color and has a dominance coefficient of -1.00 , indicating complete dominance for the pigmented allele. The median estimated location of the activity QTL listed in Table II was $50 \mathrm{cM}$. The $52-\mathrm{cM}$ albino locus estimate was within the $95 \%$ CI of estimated locations of all phenotypes in Table II except the LD2 activity measures.
The discrepancy between the open field and other test results thus appears to be a specific effect of extreme light aversiveness of the open field to albino mice. Coat color differences in activity levels support this conclusion. Albino mice are significantly $(p<0.0001)$ less active than pigmented mice in both open-field test sessions, with standardized mean differences $(\boldsymbol{d})$ of -.57 and -.41 , respectively, for first and second test sessions. In contrast, albino mice are more active in all other test apparatus $(\boldsymbol{d}=+.11$ to $\boldsymbol{d}=+.50$,

Table IV. LOD Scores and Direction of Effect of QTLs Influencing Latency to Act

\begin{tabular}{|c|c|c|c|c|c|c|c|c|}
\hline \multirow{4}{*}{$\begin{array}{l}\text { Location cM (15th-85th percentile) } \\
95 \% \text { CI of highest LOD trait }\end{array}$} & \multicolumn{8}{|c|}{ Chromosome } \\
\hline & 1 & 4 & 7 & 8 & 14 & 15 & 18 & $\mathrm{X}$ \\
\hline & $74-80$ & - & $50-54$ & - & $14-18$ & $21-26$ & $16-34$ & - \\
\hline & $70-90$ & - & $51-57$ & - & $13-23$ & $21-27$ & $20-44$ & - \\
\hline OF1: latency to enter center & & & $4.3+$ & & & $9.0+$ & $3.0+$ & \\
\hline OF2: latency to enter center & & & $17.5+$ & & & $9.8+$ & $3.2+$ & \\
\hline LD1: log latency to enter light side & $4.9+$ & & & & $11.4-$ & $19.6+$ & $3.4+$ & \\
\hline LD2: log latency to enter light side & $5.1+$ & $2.9+$ & & & $5.2-$ & $12.3+$ & $4.0+$ & \\
\hline PL: latency to enter an open arm & & & & & & $10.5+$ & & \\
\hline PL: latency to enter any arm & & & & & & $12.5+$ & & \\
\hline PL: $\log$ latency to enter 2 nd arm & & $2.8+$ & & & & $12.4+$ & $3.0+$ & \\
\hline SQ: latency to enter an open arm & & & & & & $3.0+$ & $3.7+$ & \\
\hline SQ: $\log$ latency to enter $2 \mathrm{nd}$ arm & & & & & & $10.6+$ & $3.3+$ & \\
\hline MR: $\log$ latency to enter mirror room & & & $2.8+$ & & & $17.7+$ & & \\
\hline
\end{tabular}


Table V. LOD Scores and Direction of Effect of QTLs Influencing Autonomic Behavior

\begin{tabular}{|c|c|c|c|c|c|c|c|c|}
\hline \multirow{4}{*}{$\begin{array}{l}\text { Location cM (15th-85th percentile) } \\
95 \% \text { CI of highest LOD trait }\end{array}$} & \multicolumn{8}{|c|}{ Chromosome } \\
\hline & 1 & 4 & 7 & 8 & 14 & 15 & 18 & $\mathrm{X}$ \\
\hline & $58-84$ & - & - & $22-48$ & $08-28$ & - & - & $0-50$ \\
\hline & $70-78$ & - & - & $12-50$ & $12-24$ & - & - & $46-54$ \\
\hline OF1: defecation, \# boli & $11.6+$ & & & & $9.5-$ & & & $5.3-$ \\
\hline OF1: urination score & $4.8+$ & & & & & & & \\
\hline OF2: defecation, \# boli & $10.7+$ & & & & $6.5-$ & & & $4.4+$ \\
\hline OF2: urination score & & & & & & & & $4.7-$ \\
\hline LD1: defecation, $\sqrt{ } \#$ boli & $5.6+$ & & & & $4.2-$ & & & $3.7-$ \\
\hline LD1: urination score & $4.9+$ & & & & & & & $9.6-$ \\
\hline LD2: defecation, $\sqrt{ } \#$ boli & $6.6+$ & & & & & & & $4.0-$ \\
\hline LD2: urination score & & & & & & & & $11.7-$ \\
\hline PL: defecation, \# boli & $7.4+$ & & & $3.7-$ & $7.1-$ & & & $9.8-$ \\
\hline PL: urination score & $4.5+$ & & & $3.7-$ & & & & $4.7-$ \\
\hline SQ: defecation, \# boli & $5.4+$ & & & $3.3-$ & $3.3-$ & & & $9.2+$ \\
\hline SQ: urination score & $5.1+$ & & & & & & & $7.6+$ \\
\hline MR: defecation, $\sqrt{ } \#$ boli & $3.3+$ & & & & & $3.4+$ & $3.3+$ & $5.6-$ \\
\hline
\end{tabular}

$p<0.05$ to $p<0.0001)$, and in home cages $(\boldsymbol{d}=+.18$, $p<0.005)$, as measured by motion detectors. Despite the higher total activity levels of albino mice in tests other than the open field, their proportion of time spent and activity in threatening areas of other test apparatus was equal or less than that of pigmented mice $(\boldsymbol{d}=$ +0.01 to $d=-.54$ ).

Dominance effects on behavioral phenotypes at the Ch7 QTL were consistent with coat color results. Heterozygous mice resembled the homogenous, pigmented mice, showing more open-field activity (center and total field, both test sessions) than albinos. Similarly, mean activity patterns of pigmented heterozygous and homogenous mice were nearly identical in the four other tests. Depending on the measure, both groups were either equal or less active than albinos in safe test areas, but showed a higher proportion of time and activity spent in the more anxiogenic areas of these four test environments. The pattern of LOD scores and direction of effects seen on Ch7 and QTL on other chromosomes, suggests that all areas within the open field are threatening and that OF activity measures in Table II are more appropriately grouped with phenotypes listed in Table III.

As indicated in Table II, there are consistent large Ch1 QTL effects, typically accounting for $4 \%$ to $10 \%$ of the phenotypic variance of each test's total activity measure and nearly $11 \%$ of the total activity all-test

Table VI. LOD Scores and Direction of Effect of QTLs Influencing Rearing Behavior

\begin{tabular}{|c|c|c|c|c|c|c|c|c|}
\hline \multirow[b]{2}{*}{$\begin{array}{l}\text { Location cM (15th-85th percentile) } \\
95 \% \text { CI of highest LOD trait }\end{array}$} & \multicolumn{8}{|c|}{ Chromosome } \\
\hline & $\begin{array}{c}1 \\
80-86 \\
72-88\end{array}$ & $\begin{array}{c}4 \\
26-38 \\
27-33\end{array}$ & $\begin{array}{c}7 \\
58-64 \\
56-60\end{array}$ & $\begin{array}{l}8 \\
- \\
-\end{array}$ & $\begin{array}{l}14 \\
- \\
-\end{array}$ & $\begin{array}{c}15 \\
18-24 \\
21-23\end{array}$ & $\begin{array}{c}18 \\
28-34 \\
23-37\end{array}$ & $\begin{array}{c}\mathrm{X} \\
16-42 \\
09-25\end{array}$ \\
\hline OF1: \# rears in perimeter & & $9.6-$ & $25.0-$ & & & $28.3-$ & $5.4-$ & $5.2+$ \\
\hline OF1: sec rearing in perimeter & & $3.8-$ & $14.4-$ & & & $19.3-$ & $3.6-$ & $2.8-$ \\
\hline OF2: \# rears in perimeter & & $19.3-$ & $13.8-$ & & & $24.0-$ & $7.8-$ & $7.5+$ \\
\hline $\mathrm{OF} 2$ : sec rearing in perimeter & & $7.8-$ & $7.1-$ & & & $13.3-$ & $3.6-$ & $3.1+$ \\
\hline PL: \# rears in closed arms & $3.1+$ & $8.2-$ & & & & $32.6-$ & $5.7-$ & \\
\hline PL: sec rearing in closed arms & $3.2+$ & $6.3-$ & & & & $29.1-$ & $6.9-$ & \\
\hline PL: \# rears in open arms $(\log X+1)$ & & $2.8-$ & & & & $5.0-$ & & \\
\hline SQ: \# rears in closed arms & & $4.8-$ & & & & $22.3-$ & $4.2-$ & $3.1+$ \\
\hline SQ: sec rearing in closed arms & & $3.1-$ & & & & $16.1-$ & $3.4-$ & \\
\hline SQ: \# rears in open arms $(\log X+1)$ & & $4.9-$ & & & & $25.5-$ & $4.3-$ & \\
\hline MR: \# rears in alleys & $6.5+$ & $3.4-$ & $4.3-$ & & & $36.8-$ & $4.8-$ & $3.6+$ \\
\hline MR: sec rearing in alleys & $5.1+$ & $3.1-$ & $5.2-$ & & & $40.5-$ & $5.6-$ & $5.5+$ \\
\hline
\end{tabular}


Table VII. LOD Scores and Estimated Location of QTLs Influencing General Behavior Patterns Assessed Across Multiple Tests

\begin{tabular}{|c|c|c|c|c|c|c|c|c|}
\hline & \multicolumn{8}{|c|}{ Chromosome } \\
\hline & 1 & 4 & 7 & 8 & 14 & 15 & 18 & $\mathrm{X}$ \\
\hline 1. Average total locomotor activity ${ }^{a}$ & $\begin{array}{c}\text { 31.9- } \\
76^{b}\end{array}$ & $\begin{array}{c}10.0- \\
36\end{array}$ & $\begin{array}{c}11.6+ \\
32\end{array}$ & $\begin{array}{c}4.7+ \\
60\end{array}$ & $\begin{array}{c}2.3+ \\
-\end{array}$ & $\begin{array}{c}\text { 14.4- } \\
22\end{array}$ & $\begin{array}{c}14.4- \\
28\end{array}$ & $\begin{array}{c}4.6+ \\
26\end{array}$ \\
\hline 2. Average safe area activity (excluding OF) & $\begin{array}{c}\mathbf{2 5 . 4}- \\
78\end{array}$ & $\begin{array}{l}\mathbf{7 . 3 -} \\
36\end{array}$ & $\begin{array}{c}16.7+ \\
46\end{array}$ & $\begin{array}{c}\mathbf{5 . 2 +} \\
58\end{array}$ & $\begin{array}{c}0.7+ \\
-\end{array}$ & $\begin{array}{c}11.2- \\
20\end{array}$ & $\begin{array}{c}14.0- \\
28\end{array}$ & $\begin{array}{c}4.5+ \\
50\end{array}$ \\
\hline 3. Average safe activity (all tests) & $\begin{array}{c}35.4- \\
76\end{array}$ & $\begin{array}{c}\mathbf{1 0 . 2}- \\
36\end{array}$ & $\begin{array}{c}\mathbf{6 . 0 +} \\
26\end{array}$ & $\begin{array}{c}\mathbf{6 . 0 +} \\
56\end{array}$ & $\begin{array}{c}1.2+ \\
-\end{array}$ & $\begin{array}{c}13.6- \\
20\end{array}$ & $\begin{array}{c}\mathbf{1 5 . 6}- \\
30\end{array}$ & $\begin{array}{c}\mathbf{5 . 3 +}+ \\
50\end{array}$ \\
\hline 4. Average $\%$ activity in threatening areas ${ }^{c}$ & $\begin{array}{l}7.7- \\
82\end{array}$ & $\begin{array}{l}\mathbf{6 . 1 -} \\
40\end{array}$ & $\begin{array}{c}\text { 18.4- } \\
56\end{array}$ & $\begin{array}{c}1.0- \\
-\end{array}$ & $\begin{array}{c}3.7+ \\
14\end{array}$ & $\begin{array}{c}\text { 16.4- } \\
22\end{array}$ & $\begin{array}{l}7.8- \\
34\end{array}$ & $\begin{array}{c}2.6+ \\
-\end{array}$ \\
\hline 5. Scans over open runways (PL and SQ) & $\begin{array}{l}3.6- \\
82\end{array}$ & $\begin{array}{c}\mathbf{5 . 0 -} \\
38\end{array}$ & $\begin{array}{l}7.1- \\
66\end{array}$ & $\begin{array}{c}1.6+ \\
-\end{array}$ & $\begin{array}{c}1.2+ \\
-\end{array}$ & $\begin{array}{c}\text { 18.8- } \\
20\end{array}$ & $\begin{array}{c}\mathbf{5 . 6}- \\
30\end{array}$ & $\begin{array}{c}2.9- \\
-\end{array}$ \\
\hline 6. Open arm counts on total cts (PL and SQ) $)^{d}$ & $\begin{array}{l}0.6+ \\
-\end{array}$ & $\begin{array}{c}1.3+ \\
-\end{array}$ & $\begin{array}{c}\text { 18.8- } \\
56\end{array}$ & $\begin{array}{c}1.0- \\
-\end{array}$ & $\begin{array}{c}1.6+ \\
-\end{array}$ & $\begin{array}{c}5.2- \\
22\end{array}$ & $\begin{array}{c}0.5+ \\
-\end{array}$ & $\begin{array}{c}1.6- \\
-\end{array}$ \\
\hline 7. Average \# rears (OF, PL, SQ, MR) & $\begin{array}{l}0.7- \\
-\end{array}$ & $\begin{array}{c}14.9- \\
36\end{array}$ & $\begin{array}{c}9.9- \\
64\end{array}$ & $\begin{array}{c}0.4+ \\
-\end{array}$ & $\begin{array}{c}0.8+ \\
-\end{array}$ & $\begin{array}{c}\mathbf{5 6 . 8}- \\
22\end{array}$ & $\begin{array}{c}\mathbf{1 0 . 1}- \\
30\end{array}$ & $\begin{array}{c}6.0+ \\
16\end{array}$ \\
\hline 8. Average log latency to enter new area & $\begin{array}{l}4.3+ \\
76\end{array}$ & $\begin{array}{c}\text { 3.9+ } \\
50\end{array}$ & $\begin{array}{c}0.9+ \\
-\end{array}$ & $\begin{array}{c}1.1+ \\
-\end{array}$ & $\begin{array}{c}8.9- \\
14\end{array}$ & $\begin{array}{c}\text { 35.5+ } \\
22\end{array}$ & $\begin{array}{c}8.7+ \\
30\end{array}$ & $\begin{array}{c}1.7- \\
-\end{array}$ \\
\hline 9. Average defecation score & $\begin{array}{c}\mathbf{1 5 . 8 +} \\
74\end{array}$ & $\begin{array}{c}3.1+ \\
34\end{array}$ & $\begin{array}{c}0.7- \\
-\end{array}$ & $\begin{array}{c}4.3- \\
42\end{array}$ & $\begin{array}{c}10.7- \\
24\end{array}$ & $\begin{array}{c}1.4+ \\
-\end{array}$ & $\begin{array}{c}0.7+ \\
-\end{array}$ & $\begin{array}{c}\mathbf{1 0 . 7}- \\
50\end{array}$ \\
\hline 10. Average urination score & $\begin{array}{l}9.6+ \\
76\end{array}$ & $\begin{array}{c}2.4+ \\
-\end{array}$ & $\begin{array}{c}1.3- \\
-\end{array}$ & $\begin{array}{c}5.9- \\
48\end{array}$ & $\begin{array}{c}2.0- \\
-\end{array}$ & $\begin{array}{c}1.2- \\
-\end{array}$ & $\begin{array}{c}1.8+ \\
-\end{array}$ & $\begin{array}{r}\mathbf{1 7 . 9}- \\
00\end{array}$ \\
\hline 11. Open-field activity/defecation D1 and D2 & $\begin{array}{c}41.7- \\
74\end{array}$ & $\begin{array}{l}7.1- \\
34\end{array}$ & $\begin{array}{c}8.6- \\
50\end{array}$ & $\begin{array}{c}2.4+ \\
-\end{array}$ & $\begin{array}{c}8.9+ \\
12\end{array}$ & $\begin{array}{c}5.8- \\
20\end{array}$ & $\begin{array}{c}3.7- \\
16\end{array}$ & $\begin{array}{c}2.2+ \\
-\end{array}$ \\
\hline 12. Anxiety composite (lines $3,4,8$, and 9) & $\begin{array}{c}\mathbf{2 4 . 3}- \\
76\end{array}$ & $\begin{array}{c}8.8- \\
36\end{array}$ & $\begin{array}{c}2.1+ \\
-\end{array}$ & $\begin{array}{c}2.3+ \\
-\end{array}$ & $\begin{array}{c}9.8+ \\
20\end{array}$ & $\begin{array}{c}\mathbf{2 6 . 4}- \\
22\end{array}$ & $\begin{array}{c}12.6- \\
30\end{array}$ & $\begin{array}{c}2.5+ \\
-\end{array}$ \\
\hline 13. Anxiety second-order principal component ${ }^{e}$ & $\begin{array}{c}21.3- \\
74\end{array}$ & $\begin{array}{c}\mathbf{1 3 . 7}- \\
36\end{array}$ & $\begin{array}{c}7.0- \\
64\end{array}$ & $\begin{array}{c}2.0+ \\
-\end{array}$ & $\begin{array}{c}\mathbf{5 . 4}+ \\
12\end{array}$ & $\begin{array}{c}\text { 32.8- } \\
20\end{array}$ & $\begin{array}{c}\mathbf{1 5 . 0}- \\
30\end{array}$ & $\begin{array}{c}4.0+ \\
32\end{array}$ \\
\hline 14. Home cage activity (beam and motion) & $\begin{array}{c}0.7+ \\
-\end{array}$ & $\begin{array}{l}\mathbf{5 . 5}- \\
52\end{array}$ & $\begin{array}{c}2.0+ \\
-\end{array}$ & $\begin{array}{c}3.0+ \\
60\end{array}$ & $\begin{array}{c}\mathbf{3 . 8}- \\
06\end{array}$ & $\begin{array}{c}2.2+ \\
-\end{array}$ & $\begin{array}{c}3.0+ \\
02\end{array}$ & $\begin{array}{c}4.9+ \\
48\end{array}$ \\
\hline
\end{tabular}

${ }^{a}$ Unless otherwise indicated, composite scores are based on average $\mathrm{z}$ scores. LODs $>3.4$ in boldface.

${ }^{b}$ Location of maximum LOD score, in $\mathrm{cM}$ from beginning of each chromosome. The location $95 \% \mathrm{CI} \approx 1 / \mathrm{LOD}$.

${ }^{c}$ Center area of open-field $\mathrm{T} 1$ and $\mathrm{T} 2$, light side of light-dark box T1, open runways of elevated plus and square mazes.

${ }^{d}$ Average standardized regression residuals of open/bright arm activity counts on total activity counts.

${ }^{e}$ First unrotated principal component was extracted from each test session, and then an unrotated principal component was extracted from the seven first-order UPC scores.

composite score shown in Table VII. In all cases, Ch1 QTL total activity measures show higher LOD scores than their safe-area activity counterpart measures in the same apparatus. Similar, but much smaller, QTL effects appear on chromosomes 4, 15, and 18. LOD scores of composite measures for these three chromosomes, shown in Table VII, indicate that, together, chromosomes 4,15 , and 18 account for about $13 \%$ of the phenotypic variance in activity in novel environments and only slightly less in low-threat areas of the test environments. The positive signs associated with LOD scores on chromosome 8 and chromosome $\mathrm{X}$ signify that, at these QTL, the alleles contributed by the high anxious (L1 and L2) parent strains operate to increase locomotor activity in novel environments.

The LOD scores of activity in home cages support our assumption that the two measurement methods are capturing somewhat different aspects of motor activity. The beam-break counts, which primarily reflect cage length traverses, show substantial LOD scores on chromosomes 4 and 8 that are consistent in direction of effect with activity levels observed in the test battery. These high LOD scores further support the hypothesis that chromosome 4 (Ch4) QTL and Ch8 QTL are influencing general locomotor activity levels in all environments. It is only on the Ch4 QTL, however, that activity scores obtained from the home cage motion detectors, which also record small movements and other nonambulatory behavior, show complete consistency with other activity measures. The highest motion detector LOD score occurs on the chromosome 11 QTL, which shows no other significant LOD scores for general locomotor activity. The QTL pattern observed for the motion detector measure suggests that high scores 
on this variable can result from high locomotor activity and/or from agitated motor behavior not necessarily involving ambulation.

\section{Activity in Threatening Areas}

Table III summarizes QTL effects on activity in the more threatening areas of each test apparatus. While the pattern of LOD scores is similar to that found in Table II, there are two notable differences. First, chromosome 8 exhibits no QTL influencing these "threat-area" activity measures, despite showing many significant LOD scores in low threatening test environments and home-cage activity. These results suggest that the major effect of Ch8 QTL is to influence general locomotor activity in all low-threat environments and that this QTL may not be influencing anxiety. The second discrepancy between Tables II and III involves chromosome 1. Although significant LOD scores appear frequently in Table III for the Ch1 QTL, these threat-area activity LOD scores are substantially smaller than those for total and safe-area activity in Table II, particularly when the measures involve the proportion of total activity occurring in anxiogenic threat areas. This difference between threat-area activity and total activity for Ch1 QTL is particularly noticeable with respect to the all-test composite measures in Table VII. The total activity composite for Ch1 QTL, with a LOD score of 32, accounts for nearly four times as much phenotypic variance as the percent activity in threat-areas composite does. In contrast, LOD scores for total activity are generally similar to LOD scores for threat-area activity for the QTL on other chromosomes. With respect to locomotor activity patterns, the influence of Ch1 QTL appears to be that of suppressing activity in most novel situations. The anxiogenic threshold for activity suppression for Ch1 QTL appears to be low, failing to appear only in the dark alleys of the mirror box apparatus.

The Ch7 QTL, which is associated with decreased total activity in the open field but slightly increased total activity in other tests, acts to suppress activity in threatareas of the two elevated mazes. In contrast, in the lightdark box retest, the Ch7 QTL increases activity in the lighted side. We believe that this increase simply reflects Ch7 QTL's effect of increasing all activity in this test, as the QTL did not increase the proportion of activity on the light side of the box. As noted below, the phenotypic data indicate that the light side of the lightdark box had lost its anxiogenic properties by the time of retest at the end of the test battery. The failure of $\mathrm{Ch} 7$ QTL to suppress differentially light-side activity in the light-dark box suggests that the relatively low light level (20 lx) of the lighted side of this apparatus was insufficient to produce sensory aversiveness in albino animals. The overall suppressive effect of the Ch7 QTL on activity in threatening areas can be seen in the four composite measures of risk activity in Table VII.

\section{Latency to Enter a Novel Area}

Table IV summarizes LOD scores of QTL that influence latency to enter initially a new area of a test apparatus. The QTL pattern for latency measures differs from that observed for total activity and for activity levels in safe and threatening areas of test apparatus. Most obvious are the consistently high LOD scores for all latency measures for chromosome 15 (Ch15) QTL, especially the z score summation across all latency measures, shown in Table VII. The Ch15 QTL shows a substantial influence on a general tendency to hesitate before entering novel environments in all of the test situations. Ch15 QTL accounts for $12 \%$ of the phenotypic variance in the composite latency measure, with the QTL, on chromosomes 1, 4, 14 , and 18 , together accounting for an additional $9 \%$. It should be noted that, despite the unambiguous and large genetic influences on what appears to be a very general Latency to Act trait, the phenotypic factor analysis summarized in Table I did not identify a general latency factor.

\section{Autonomic Behavior}

Table V summarizes QTL LOD scores for autonomic reactivity (defecation and urination) measures obtained in all tests. QTL on chromosomes 7, 15 and 18 , which exhibit many substantial LOD scores for activity and latency measures of anxiety, show no significant QTL for anxiety manifested by defecation and urination. Ch4 QTL also shows no significant LOD scores for the autonomic measures in individual tests, which is consistent with the interpretation that this QTL is primarily influencing general activity level rather than anxiety produced activity suppression.

\section{Rearing Behavior}

Table VI summarizes frequency and duration of rearing measured in the two elevated mazes, the mirror-chamber apparatus and in the perimeter of the open field. Note that the automated detection system did not distinguish between rearing associated with grooming and other rearing-related behaviors. Most 
salient in Table VI is the extremely high LOD scores on Ch15 QTL for all rearing measures except open runway rears in the plus maze, a very low-frequency behavior. Consistent patterns of suppression of rearing are also seen on Ch4 and Ch18 QTL, but of considerably lower magnitude than that on Ch15.

\section{Composite Measures}

As previously indicated, Table VII summarizes LOD scores of composite measures assessed across two or more different tests. These composite scores reduce the influences of idiosyncratic characteristics of specific test environments and thus reflect more general behavioral traits than do the individual component measures. LOD scores of these composite measures are well above the median LOD scores of their individual components, reflecting the consistency of QTL effects on these behavior patterns across tests. The highest LOD score is for the composite measure of rearing, (56.8) on chromosome 15 , which explains $19 \%$ of the phenotypic variance.

Table VII is useful for interpreting the nature of an individual QTL's effect on behavioral traits related to anxiety and activity. By using several relatively independent composite measures that have high LOD scores on a chromosome, one can approximate the location of the QTL on that chromosome. For example, using QTL for composite traits 3, 9, and 11 for chromosome 1 and traits 5, 7, and 8 for chromosome 15, we obtain the following highly overlapping $95 \%$ CI for location estimates:

Ch 1, safe activity, excluding open field: $74-78 \mathrm{cM}$

Ch 1, average defecation score: $71-77 \mathrm{cM}$

Ch 1, open field activity/defecation composite: $73-75 \mathrm{cM}$

Ch 15 , scans over open runways: $17-23 \mathrm{cM}$

Ch 15 , average number of rears: $21-23 \mathrm{cM}$

Ch 15, latency to enter new area: $21-23 \mathrm{cM}$

Similar high-consistency patterns can be seen for chromosomes 4 and 18 . There is no evidence of widely separated multiple QTL on any of these chromosomes. Because precision in location estimates is a function of (LOD scores $)^{-1}$, consistency of multiple estimates is lower for QTL with smaller observed effects on other chromosomes. Although the QTL positions are widely separated, suggesting that there are at least two QTL on these chromosomes, we have not been able to distinguish the action of a single pleiotropic locus from multiple QTL (Aguilar et al., 2002; Knott and Haley, 2000).

Table VIII provides the phenotypic correlations among the across-test general behavior patterns listed in Table VII. Within the test battery, increased defecation scores are associated with less total activity, longer latencies to enter new test areas, and proportionally lower activity in anxiogenic areas of the different test apparatus, a pattern consistent with an interpretation of

Table VIII. Phenotypic Correlations Among General Behavior Patterns Assessed Across Tests

\begin{tabular}{|c|c|c|c|c|c|c|c|c|c|c|c|c|}
\hline & \multicolumn{12}{|c|}{ Composite Measure } \\
\hline & 2 & 3 & 4 & 5 & 6 & 7 & 8 & 9 & 10 & 11 & 12 & 13 \\
\hline 1. Average total locomotor activity ${ }^{a}$ & .91 & .94 & .40 & -.41 & -.03 & .38 & -.53 & -.21 & -.07 & .41 & .74 & .11 \\
\hline 2. Average safe area activity (all tests) & & .94 & .48 & .40 & -.04 & .43 & -.52 & -.24 & -.09 & .59 & .78 & .16 \\
\hline 3. Average safe area activity (excl. OF) & & & .30 & .36 & -.12 & .32 & -.45 & -.19 & -.08 & .38 & .69 & .11 \\
\hline 4. Average $\%$ activity in threatening areas ${ }^{b}$ & & & & .59 & .60 & .54 & -.63 & -.17 & .01 & .55 & .75 & .09 \\
\hline 5. Scans over open runways (PL and SQ) & & & & & .64 & .45 & -.44 & -.08 & .05 & .26 & .52 & .08 \\
\hline 6. Open arm counts on total cts (PL and SQ) $)^{c}$ & & & & & & .22 & -.31 & .02 & .11 & .10 & .27 & .03 \\
\hline 7. Average \# rears (OF, PL, SQ, MR) & & & & & & & -.47 & -.15 & .01 & .36 & .52 & .05 \\
\hline 8. Average log latency to enter new area & & & & & & & & .19 & .07 & -.40 & -.81 & .05 \\
\hline 9. Average defecation score & & & & & & & & & .55 & -.63 & -.56 & .11 \\
\hline 10. Average urination score & & & & & & & & & & -.30 & -.25 & .16 \\
\hline 11. Open field activity/defecation composite ${ }^{d}$ & & & & & & & & & & & .70 & .06 \\
\hline 12. Anxiety composite (lines $3,4,8$, and 9) & & & & & & & & & & & & .01 \\
\hline 13. Home cage activity (beam and motion) & & & & & & & & & & & & - \\
\hline
\end{tabular}

${ }^{a}$ Unless otherwise indicated, composite scores are based on average z scores. Significance of correlations: $p<0.01$ when $r>0.06$; $p<0.001$ when $r>0.08$.

${ }^{b}$ Center area of open-field T1 and T2, light side of light-dark T1 and T2, open runways of elevated plus and square mazes.

${ }^{c}$ Average standardized regression residuals of open arm activity counts on total activity counts.

${ }^{d}$ Pooled z scores, activity and defecation (signs reversed), sessions 1 and 2. 
Table IX. Test 2 - Test 1 Differences in Open Field and in Light-Dark Box Phenotypic Scores and Significant LOD Scores of T2-T1 Difference Measures

\begin{tabular}{|c|c|c|c|c|c|}
\hline \multirow[b]{2}{*}{ T2-T1 Difference Measure } & \multirow[b]{2}{*}{$d^{a}$} & \multirow[b]{2}{*}{ significance $(p)$} & \multicolumn{3}{|c|}{ LOD Score ${ }^{b}$} \\
\hline & & & $\mathrm{CH} 4$ & $\mathrm{CH} 7$ & $\mathrm{CH} 15$ \\
\hline \multicolumn{6}{|l|}{ Open Field } \\
\hline Total \# activity cts & +.12 & .0001 & $3.9-$ & $3.2-$ & $1.7+$ \\
\hline Activity cts in center & -.04 & .0983 & $3.6-$ & $3.3-$ & $0.5+$ \\
\hline Latency to enter center & +.06 & .0276 & $0.3+$ & $2.9+$ & $0.7-$ \\
\hline Defecation, \# boli & +.18 & .0001 & $0.5-$ & $2.9+$ & $0.6+$ \\
\hline \multicolumn{6}{|l|}{ Light-Dark Box } \\
\hline Total activity cts & +.08 & .0017 & $2.4-$ & $1.9+$ & $14.0+$ \\
\hline Activity cts on light side & +.47 & .0001 & $2.3-$ & $0.4+$ & $11.3+$ \\
\hline Latency to enter light side & -.75 & .0001 & $0.8+$ & $0.4-$ & $2.7-$ \\
\hline Defecation, \# boli & +.12 & .0001 & $1.7-$ & $2.6-$ & $2.2-$ \\
\hline
\end{tabular}

a behavior pattern reflecting fear or anxiety. Home cage activity level has a small but significant positive correlation with activity levels in the test battery, but home cage activity also correlates positively with defecation levels in the test battery.

The open-field and the light-dark box were the first two tests administered in the battery, and both were repeated at the end of the test battery. Table IX summarizes the Test 2 minus Test 1 (T2-T1) behavioral change measures in the open-field and in the light-dark box. Shown in boldface in Table IX are the only significant LOD scores of change (T2-T1) measures. Total activity and defecation increased slightly (all $\boldsymbol{d}<.2$ ) at retest in both the open-field and light-dark box. All remaining open-field measures, including those not listed, show no significant T2-T1 differences. Despite the very small overall test-retest differences in open field phenotypic behaviors, LOD scores did increase substantially on retest for total center activity measures for QTL on chromosomes 4 and 7 (Table III). The slight general increase in open-field total activity at retest appears to be suppressed by the QTL on chromosomes 4 and 7, probably for different reasons, because Ch4 QTL largely influences general activity and Ch7 QTL appears to be related to visual effects associated with the albino locus.

Mice show much less aversiveness to the light side of the light-dark box when retested at the end of the test battery than they exhibit on initial exposure. Median latency to enter the light side of the apparatus decreased from an initial $60.2 \mathrm{~s}$ to $17.4 \mathrm{~s}$ at retest, with a $50 \%$ increase in light area entries and a $33 \%$ increase in light area activity in the second test session. Given that the illumination level of the light side of the lightdark box is relatively low, and similar to the illumination levels of the elevated mazes, adaptation is not surprising. Large light-dark Test 1 LOD scores for light activity and total activity on Ch15 QTL drop to insignificance on Test 2. The LOD scores for the T2-T1 difference measures, shown in Table IX, indicate a strong directional QTL influence toward increasing both light area activity and total activity at retest. If the phenotypic data indicate that the light side of the lightdark apparatus is no longer anxiogenic to mice during retest, the disappearance of a Ch15 QTL effect on T2 light- and total-activity would be expected, because the Ch15 QTL effect on activity primarily involves suppression of movement in anxiogenic environments. In contrast, Ch1 QTL effects on T1 and T2 sessions are comparable for light area and total activity measures, despite the large decrease in latency to first enter the light area. This T1-T2 QTL consistency further supports the view that, unlike the Ch15 QTL, the Ch1 QTL suppression effect on activity cuts across a wide range of anxiogenic levels.

Although LOD scores for the light-activity and total-activity change measures in the light-dark box are large, Appendix Tables I and II show that there are few T1-T2 change measure LOD scores exceeding 3.0. A comparison of LOD scores for Test 1 and Test 2 sessions in the open field (Appendix Table I) shows that the interpretation of QTL effects is highly similar for the two sessions, despite the extensive testing and handling of mice that occurred between T1 and T2. 
Although T1 and T2 QTL for the light-dark box were less consistent than the open-field results, with the exception of the Ch15 QTL T1-T2 differences described above, results for the two widely separated test sessions were largely comparable. Other than the light-dark box activity T1-T2 dissimilarity, the differences between Test 1 and Test 2 phenotypic means and variances on other measures, although sometimes significant, were small in magnitude. Similarly, while occasionally significant, the magnitude of Sex by Test Session interaction effects tended to be trivially small for all open-field arena and light-dark box repeated measures.

Figure 1 displays the genome scans for the two replicate F2 intercrosses for five composite measures from Table VII that best capture the four dimensions of anxiety detected in this paper (lines 3, 4, 7, 8, and 9). The high consistency between the two replicates (G1 and G2) is evident for all five dimensions. One quantitative index of this consistency is the correlation between LOD scores obtained in the two replications, for a given phenotype, taken every $10 \mathrm{cM}$. These ranged from .64 for safe activity to .95 for rearing. Note, however, that LOD score distributions are highly skewed.

Although the phenotypic factor analysis summarized in Table I largely identified test session factors, we also computed LOD values for the 11 factor scores obtained from the analysis, to compare to LODs obtained from individual measures and the five composite dimensions captured in Table VII. These factor LOD scores are shown in Table X. As would be expected, QTL showing large effects for individual behavioral measures showed comparable effects for factor scores. At issue, however, is whether the genetic dissection of phenotypic factor scores is comparable or superior to using individual measures and their logically derived composites. This issue is addressed below.

\section{DISCUSSION}

We have presented genetic mapping data on over 100 phenotypes collected on a battery of measures that putatively measure anxiety in rodents. A total of 17 QTL were detected, which together accounted for $20 \%$ to $36 \%$ of the phenotypic variance of each of our composite measures of anxiety. Typically, four to six QTL influence each measure, with the largest QTL explaining no more than half of the variance attributable to detected QTL. A frequency distribution of QTL effect sizes across all 20 chromosomes is shown in Figure 2. Approximately $1 / 5$ of the examined QTL influenced at least $2 \%$ of the phenotypic variance in these five dimensions of anxiety. However, each of the five composite measures is influenced by at least one QTL that accounts for over $5 \%$ of its phenotypic variance, and most of the genetic variance for each measure is due to QTL with effect sizes greater than $1 \%$.

The results we have obtained extend those previously reported and are discussed first. We then turn to consider the behavioral dimensions that we can identify, and finally turn to consider issues of behavioral assessment.

\section{Profiles of QTL Action}

Consistent with previous findings with $\mathrm{F} 2$ mice from these strains, QTL on chromosomes 1 and 15 show the largest effects on behavioral manifestations of anxiety (Flint et al., 1995, Turri et al., 2001b). The major influence of the QTL on chromosome 1 is to

Table X. LOD Scores and Direction of Effect of QTLs Influencing Phenotypic Factor Scores for the 11 Factors in Table I

\begin{tabular}{|c|c|c|c|c|c|c|c|c|}
\hline \multirow[b]{2}{*}{ Factor } & \multicolumn{8}{|c|}{ Chromosome } \\
\hline & 1 & 4 & 7 & 8 & 14 & 15 & 18 & $\mathrm{X}$ \\
\hline 1 & $6.3-$ & & $13.2-$ & & & $8.4-$ & $6.3-$ & \\
\hline 2 & $3.6-$ & $9.7-$ & $25.2-$ & & & $8.2-$ & $5.8-$ & $3.4+$ \\
\hline 3 & $8.5-$ & & $3.5+$ & & & $8.7-$ & $7.1-$ & \\
\hline 4 & & $3.2-$ & $13.1-$ & & $3.0+$ & $8.8-$ & & $4.0-$ \\
\hline 5 & $5.5-$ & $4.3-$ & $6.1-$ & & $2.9+$ & $20.4-$ & $6.7-$ & $4.9+$ \\
\hline 6 & $17.6-$ & $3.4-$ & & & $7.4+$ & $14.0-$ & $5.0-$ & \\
\hline 7 & $10.4-$ & $9.2-$ & $3.0+$ & & & & $5.6-$ & $3.8+$ \\
\hline 8 & & $7.6-$ & $3.3+$ & & & $6.8-$ & $5.9-$ & \\
\hline 9 & $8.3+$ & $3.6-$ & $4.3-$ & & & $44.9-$ & $6.1-$ & $3.7+$ \\
\hline 10 & $36.8+$ & $3.2+$ & $7.6-$ & $9.9-$ & & & $3.5+$ & $6.8-$ \\
\hline 11 & $15.5+$ & & & $5.5-$ & $7.7-$ & & & $21.1-$ \\
\hline
\end{tabular}



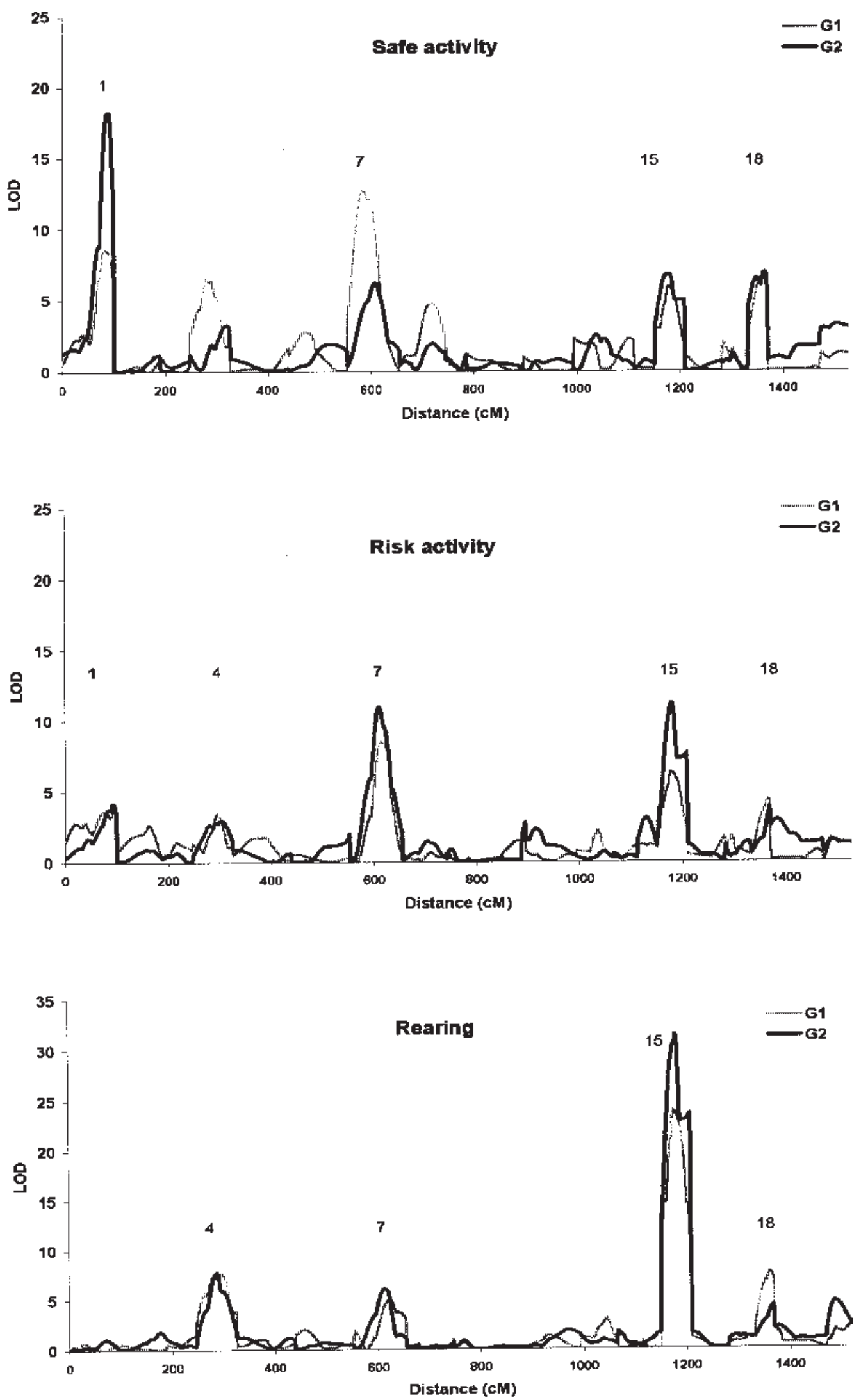

Fig. 1. Genome scans for five composite dimensions of anxiety for the two replicate F2 intercrosses, G1 and G2. LOD scores for the pooled replicates are approximately the sum of the individual replicate LOD scores. Correlations between G1 and G2 LOD scores taken every $10 \mathrm{cM}$ were: .64 (safe activity), .81 (risk activity), .95 (rearing), .92 (latency), and .71 (defecation). Note, however, that LOD score distributions are all highly skewed. End boundaries (cM) for chromosomes one through X, respectively, are: 98, 188, 246, 324, 438, 552, 654, 744, 784, 894, 992, 1064, 1110, 1150, 1208, 1280, 1330, 1368, 1470, 1526. Chromosomes with significant QTL effects are labeled. 

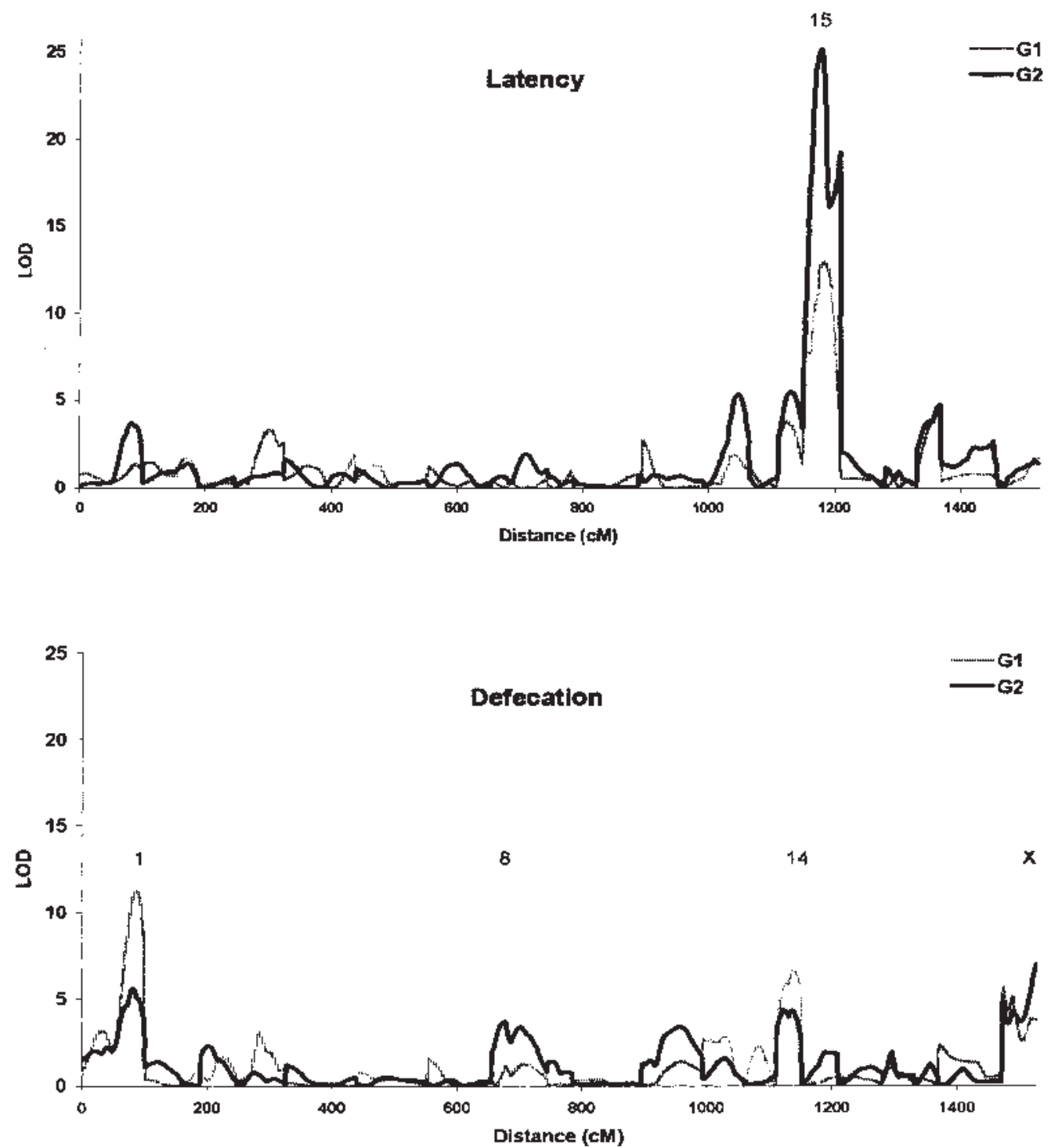

Fig. 1. Continued

reduce activity throughout all areas of each test apparatus, with somewhat greater suppression of activity in the more anxiogenic areas, and to increase autonomic reactivity during testing. In all but the mirror-box test, gene action appeared to be partially dominant, with mice heterozygous for the Ch1 QTL typically behaving like the low-active, high defecating, homozygous mice.

The QTL on chromosome 15 differs from Ch1 QTL in its pattern of influence in several ways. First, although Ch15 QTL also suppresses activity in moderately anxiogenic environments, the effect is considerably weaker than that for Ch1 QTL. In contrast, the differential suppression of activity in higher anxiogenic areas of the test apparatus, particularly in the elevated plus and square mazes, is larger for Ch15 QTL than for Ch1 QTL. Second, Ch15 QTL has a large effect on increasing latencies of mice to move from one area of a test apparatus to another. This QTL, which accounted for $12 \%$ of the variance in composite latency scores, shows consistent effects on all latency measures in all tests, compared to a significant but small overall effect for Ch1 QTL. Similarly, the Ch15 QTL has a large and consistent effect on suppressing rearing behavior in all tests, accounting for $19 \%$ of the phenotypic variance in the composite, whereas Ch1 QTL has only minor and environmentally limited influences on rearing. There is also a complete absence of a Ch15 QTL influence on 


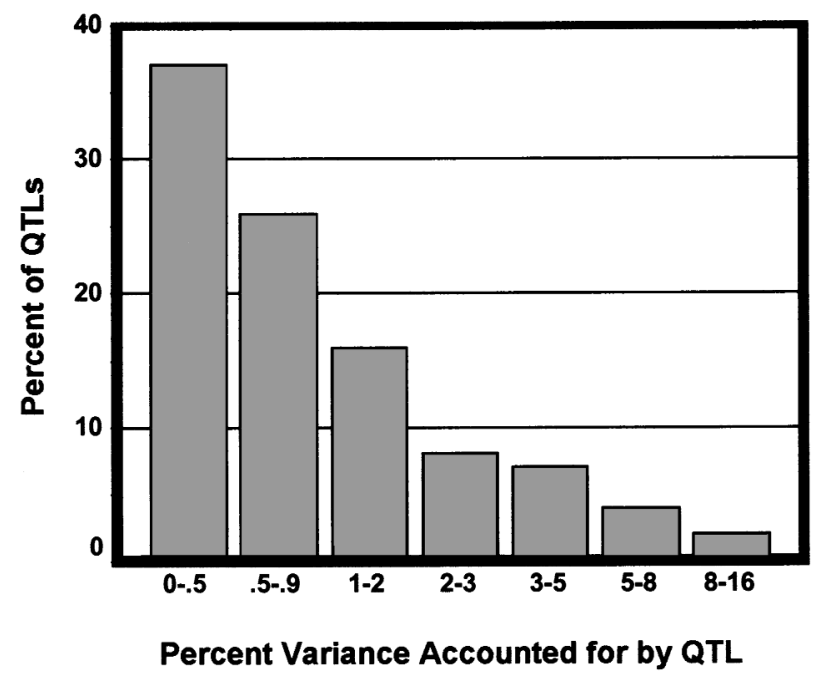

Fig. 2. Percentage of phenotypic variance in five composite scores accounted for by the 17 QTL examined. QTL accounting for less than one percent of the phenotypic variance are nonsignificant.

autonomic activity, whereas the Ch1 QTL effect is large. Finally, gene action on Ch15 QTL is additive, in contrast to the partial dominance seen on chromosome 1.

The profile of Ch18 QTL influences is similar to that of Ch15 QTL, but with less pronounced effects on the suppression of activity in high anxiogenic areas, latency measures, and rearing. The QTL on chromosomes 4,8 , and $\mathrm{X}$ all appear to influence general locomotor activity in both home cages and in the test battery, but the three QTL effects differ with respect to differential suppression of activity in high- and lowanxiogenic test areas and autonomic reactivity. Ch14 QTL has a consistent influence on latencies, autonomic reactivity, and avoidance of anxiogenic regions, but shows no substantive influence on general activity levels.

The QTL on chromosome 7 presents a more complicated picture than other QTL. As suggested above, Ch7 QTL is likely to involve the albino (tyr) locus, or be closely linked to it, because the QTL is a nearly perfect predictor of recorded albino/pigmented coat color, and thus reflects the action of the tyrosinase mutation on behavior (Collins, 1964, DeFries, 1969). Behavioral phenotypes of pigmented heterozygous and homozygous mice are largely indistinguishable. The low-activity parent alleles of the Ch7 QTL exhibit higher total locomotor activity in the four dimly lit tests and in home cages, yet both time spent and activity are reduced the in open runways of the two elevated mazes and in the center of the open field, suggesting a strong wall hugging response of albino animals. Because the Ch7 QTL has no substantive effect on latency scores or autonomic reactivity in tests other than the open field, its influence on reducing activity in the nonwalled sections of tests is at least partly a visually mediated effect. Clearly, however, all relevant open-field measures indicate that albino mice exhibit greater anxiety than both homozygous and heterozygous pigmented mice in this test. Furthermore, there is no adaptation to the open field upon retest, also indicating that bright light is highly aversive to albino mice. All measures indicate that the open field is more stressful to albino mice than pigmented mice, resulting in greater anxiety among albinos. We contend that the anxiety shown by albino mice in the open field can be described as "state anxiety," resulting from a specific environmental stimuli $x$ visual system interaction. Our results provide a multitest extension of early work, which used an F5 generation from the progenitor strains of our current F2 mice. DeFries et al. (1966) found that large open-field activity differences between albino and pigmented mice disappeared when testing was done under red light. They concluded that there is a major gene effect on openfield activity mediated by the visual system and that albino mice are more photophobic than pigmented mice under conditions of bright illumination.

The suppression of all forms of open-arm activity and edge scanning in the two elevated mazes would also normally be regarded as indicative of anxious behavior in mice. In the case of albino mice, however, we must alternatively consider that avoidance of open runways reflects a simple behavioral adaptation by visually impaired animals, not necessarily involving an emotional component. A nonanxiety interpretation of open-runway behavior is supported by the lack of $\mathrm{Ch} 7$ QTL effects on the many other measures of anxiety assessed in the two elevated mazes and in the light-dark and mirror-chamber boxes. Several previous studies of mouse anxiety, failed to detect a Ch7 QTL effect (Flint et al., 1995; Gershenfeld et al., 1997; Gershenfeld and Paul, 1997). We suggest that this may be due to two factors. First is the lower power of these earlier studies, with smaller Ns and genotyping of only extreme behavioral phenotypes, which greatly increases the chances of false negatives (Flint, 2003). Second is the opposing effects of the Ch7 QTL-increased activity in safe areas but substantial suppression of activity in bright areas and open runways-which may counteract each other in some test situations.

\section{Principal Components}

Principal components analysis has often been used to extract commonalities from tests as a way of 
obtaining a relatively unconfounded measure of anxiety. Consideration of the relative contribution from individual measures to extracted factors is the way factors are then interpreted. Much of this previous work has been reviewed by Ramos and Mormede (1998). One can also compute unrotated first principal components from each test session, for use as global measures of anxiety in various test environments. Appendix Tables I through IV show these first unrotated PCs for four tests in the present battery. These within-test principal components are much less informative than the original phenotypic measures obtained from a powerful genetic design.

In Table X, we use genetic effects to interpret the 11 full-battery phenotypic factors described in Table I, by examining the number and size of QTL that influence the factors. Our results show that the factor analysis and subsequent QTL mapping of obtained factor scores adds little to what emerges from the analysis of individual measures and the construction of composite measures. QTL mapping of the eight test-specific factors identified the same loci that influence the individual measures of each test, with LOD scores similar to those associated with the individual measures. The phenotypic factor analysis also failed to identify a factor for latency and factors that could be said to represent activity in safe and threatening environments. Genetic mapping of phenotypic factors identified the same loci that influence variation in individual behavioral measures but did little to define loci that influence anxiety. In sum, phenotypic factor analysis (i) did not obviate the need to consider individual scores, (ii) generated factors that were inferior to simple cross-test composite scores of similar behaviors, (iii) failed to identify any novel loci, and (iv) did not make the interpretation of the genetic effects any more transparent.

It is worth considering why a principal components analysis of multitest measures was unsuccessful. The phenotypic variance-covariance $(V-C)$ matrix on which correlation coefficients are based represents the sum of three $V$ - $C$ matrices-the genetic $V$ - $C$ matrix, the environmental history $V$ - $C$ matrix, and the test session $V$ - $C$ matrix. It is the latter, test session $V$ - $C$ matrix, that creates a problem in animal research using test batteries. Idiosyncratic environmental events that occur just before or during a test can have a large effect on the variances and covariances of measures obtained in that specific test session, but will have no effect on covariances between those test measures and measures obtained in other tests. As a result, phenotypic correlations within test sessions tend to be larger than between-test correlations and the resulting factor analysis tends to produce test session factors that obscure subtle cross-test behavioral consistencies. Because idiosyncratic test environment effects are averaged out when cross-test composite scores are used, the phenotypic correlations among composites such as those shown in Table VIII, more heavily reflect genetic and environmental history covariance than do individual measures.

When inbred strain means replace individual subject observations, (e.g., Collins et al., 1988, Henderson, 1979; Ramos and Mormede, 1998; Trullas and Skolnick, 1993) the influence of test session and environmental history $V-C$ matrices are reduced by a factor of ( $n$ per strain $)^{-1}$, effectively creating a genotypic correlation matrix when $n$ is even moderately large. Factor analyses of genotypic correlation matrices thus avoid the confounding influences of test session environmental effects. Because the number of strain means tends to be low relative to the number of dependent variables analyzed, genotypic factor analyses have traditionally been viewed with caution. Recent work showing that the ratio of variables to observations is not the critical element in the stability of factor analytic solutions (Preacher and MacCallum, 2002) indicates that these genotypic factor solutions can often be quite stable. At each locus in the current F2 genetic analysis, our sample of 1636 is divided into three genotypes, with the smallest group having a sample size in excess of 400. Consequently, the influence of the test session $\mathrm{V}$-C matrix on the phenotypic $\mathrm{V}$-C matrix is reduced by a factor of at least $1 / 400$, thus trivializing these test environment influences in our study.

\section{Composite Measures}

Our data can be captured in five broad behavioral dimensions, as in the five lines within Table VII. These dimensions are:

(i) Suppression of activity in moderately anxiogenic "safe areas" of test environments outside of the home cage (Line 3)

(ii) Tendency to avoid the more anxiogenic areas of test environments (Line 4)

(iii) Suppression of rearing behavior (Line 7)

(iv) Hesitancy to enter novel areas of test environments (Line 8)

(v) Autonomic reactivity in novel environments (Line 9)

QTL identified through analysis of individual test measures are found to influence at least two of these anxiety dimensions. Furthermore, there is often a 
consistency in the paired direction of effects across the QTL, signifying the presence of genetic correlations among these five traits. The QTL consistency is high for activity and autonomic reactivity, suggesting a large negative genetic correlation between these traits, a finding in agreement with open field data for these two measures (Hegmann and DeFries, 1968, Henderson, 1989). A similar, but weaker, QTL consistency exists between anxiogenic-area "risk" activity and latency measures, suggesting a moderate negative genetic correlation between these two dimensions. The QTL pattern of influence on rearing behavior is also more consistent with the latency and percentage of risk activity dimensions than it is with general activity measures. Thus, while our results support the conclusions of van Abeelen et al. (1973) that rearing and locomotion are genetically correlated in a positive direction, our data suggest that this relationship may be limited to situations in which activity is measured in anxiogenic environments.

The much lower consistency of QTL effects for latency and autonomic reactivity, suggests that a low, possibly zero, genetic correlation exists between these two traits. This independence of the autonomic reactivity and latency-to-act dimensions is consistent with Gray's distinction between the flight-fight (autonomic) and behavioral inhibition (latency) systems operating in response to threatening stimuli (Gray, 1987; Gray and McNaughton, 2000). Barlow (2000) made a similar distinction between a negative affective state and a strong physiological component of human anxiety. Because no two traits appeared together across all major QTL, these behavioral dimensions, although correlated, appear to be genetically separable manifestations of anxiety in mice. What cannot be ascertained from the current data is whether a single QTL at one chromosomal location accounts for the influences on multiple traits or if these influences are due to closely linked QTL specific for each trait.

\section{Assessment of Behavioral Phenotypes}

Considerable time and effort has been invested in evaluating the relative merits of various test apparatus, testing procedures, and specific measures within tests with respect to assessing anxiety in rodents. Judgments concerning the efficacy of various measures and procedures have often been based on subjective, rather than statistical, comparisons of effect sizes of different measures, often in studies with small numbers of animals. Consequently, there is a wide diversity of opinions regarding the measurement of anxiety in rodents
(Belzung, 2001; Belzung and Le Pape, 1994; Costall et al., 1989; Crawley, 1985; Hogg, 1996; Rodgers, 1997; Rodgers and Cole, 1994; Toubas et al., 1990). The current study, with 1636 mice and 17 QTL to serve as independent variables, provides an opportunity to compare directly, with some precision, approximately 100 behavioral measures that have been considered manifestations of anxiety in rodents.

First, although the number of behavioral phenotypes obtained from each test apparatus differed substantially, all tests produced multiple measures that showed highly significant (LOD > 10) QTL effects. Furthermore, there was a consistency of results across all tests. We see no compelling evidence to suggest the overall superiority of any single test apparatus in the battery. Because the nature and intensity of anxiogenic environments differs in each test apparatus, there is a strong case for using a battery of tests for assessing anxiety to help disentangle sensory and motor elements from anxiety based responses, such as the Ch7 QTL effects described above. By providing a graded range of anxiogenic environments, differing test environments also provide an opportunity for a psychometric approach to interpreting the behaviors observed, as described below.

Second, other than a tendency for measures of time spent in various areas/activities to have somewhat lower LOD scores than their comparable frequency count measures, and for defecation LOD scores to usually exceed urination LOD scores, there were few instances in which one measure within an apparatus produced consistently larger effect sizes than commonly used alternative measures of the same behavior. In general, ratio scores, regression residuals and other composites of two measures (e.g., percentage activity counts in open arms, arena center, etc.) produced lower LOD scores than component measures, as would be expected from the compounding of measurement error in such composites. Nevertheless, these composites often aided in interpretation of behavior patterns within a specific test and provided directly comparable measures across tests.

Third, the test battery used in our experiment proved to be highly effective in identifying QTL. Some researchers hesitate to use multiple tests, fearing that earlier tests in a battery may confound or attenuate experimental effects observed in succeeding tests. Our pilot studies, using counterbalanced orders, showed few such effects of any consequence, hence we chose a fixed order of testing in the current battery. The results speak for themselves, with respect 
to the consistency and size of the experimental effects observed throughout the test battery. Furthermore, the open-field and light-dark tests were administered both at the beginning and at the end of the test battery, with largely comparable results. Any losses in precision that may have resulted from possible treatment $\times$ prior test interactions were overshadowed by the information gain resulting from the use of the test battery.

Fourth, attempts to distinguish between activitybased and anxiety-based behavioral phenotypes in a test apparatus can be misleading. Exploration in the low anxiogenic areas of a test apparatus is often regarded as an assessment of general activity level whereas suppressed exploration in the more threatening areas is regarded as an index of anxiety level (e.g., Landgraf and Wigger, 2002). Out-of-cage test environments range from moderately anxiogenic to highly threatening; hence behaviors in all test areas are likely to be probes for anxiety in a heterogeneous population. The effects of Ch1 QTL provide a case in point: a major influence of Ch1 QTL was to suppress locomotor activity in all low threatening test areas, except the dark narrow alleys of the mirror-chamber box. The implication that the Ch1 QTL effect is involved with the suppression of general locomotor activity unrelated to anxiety is strongly counter-indicated by this QTL's consistent effects on all other indices of anxiety, but no effect on home cage activity.

It would be more fruitful to view various anxiogenic regions of different test apparatus as "test items" in the psychometric sense, each with its own continuous variation analog of an item-characteristic curve (ICC) (Lord, 1980) and that the mix of test ICCs should be appropriate for the population being studied. Activity ratio scores, such as percentage of activity in the most anxiogenic test areas, should be used cautiously and in conjunction with their component measures, because subjects showing a very low anxiogenic threshold for activity suppression will sometimes have higher proportions of activity in threatening areas than subjects with higher anxiogenic thresholds.

\section{CONCLUSIONS}

The current study demonstrates the effectiveness of genetic mapping to study a behavioral construct, such as anxiety, in rodents. The results emphasize the advantages for studying such traits by using multiple behavioral measures, a large $n$ and a full-sample genome-wide screen. Reliance on a limited test battery for the definition of a complex phenotype such as anxiety can result in the identification of genetic loci that influence confounds, such as activity or sensorymediated effects, rather than the behavior of interest. We have argued here that the reduction of multiple measures, using factor analysis, is not the most efficient process for identifying relevant loci; rather we recommend the analysis of composite measures that tap into measures common to all behavioral tests.

Our results suggest that anxiety expressed in novel environments is a complex trait, involving at least five dimensions: the suppression of activity in mildly anxiogenic environments, the suppression of activity in threatening environments, rearing, hesitancy to enter a novel area, and autonomic reactivity. All QTL detected were found to influence two or more of these dimensions, with the consistency of influences across QTL suggesting genetic correlations among the four dimensions ranging from low to high. In most cases, a small number of QTL appeared to capture most of the genotypic variance in the trait, but in no case did a single QTL account for a substantial portion of the genetic variance.

The optimal way of analyzing multiple phenotypes in a genetic mapping study is still unclear, but must depend on the intended aims. In some cases it will be important to find the best phenotype for subsequent fine-mapping and gene identification: for this purpose, our results suggest that a cross-test measure of rearing would be the most suitable measure. In other cases, multiple phenotypes could be employed for the genetic validation of a phenotype and for physiological profiling. Our results, and those of others (Stoll et al., 2001), indicate that it would be appropriate to analyze the measures individually. However, multivariate approaches are likely to increase power and can be used for tests of pleiotropy (Knott and Haley, 2000). We deal with these issues in a separate article.

\section{APPENDIX}

\section{Extended Tables of LOD Scores Obtained from Each Test Apparatus}

LOD scores of 2.0 or larger are presented for an extended set of variables for the all chromosomes, except Ch9, Ch10 and Ch19, which produced no LOD scores greater than 2.8. Each of the remaining 17 chromosomes presented in Appendix Tables I-V had at least one LOD score greater than 4.2. 


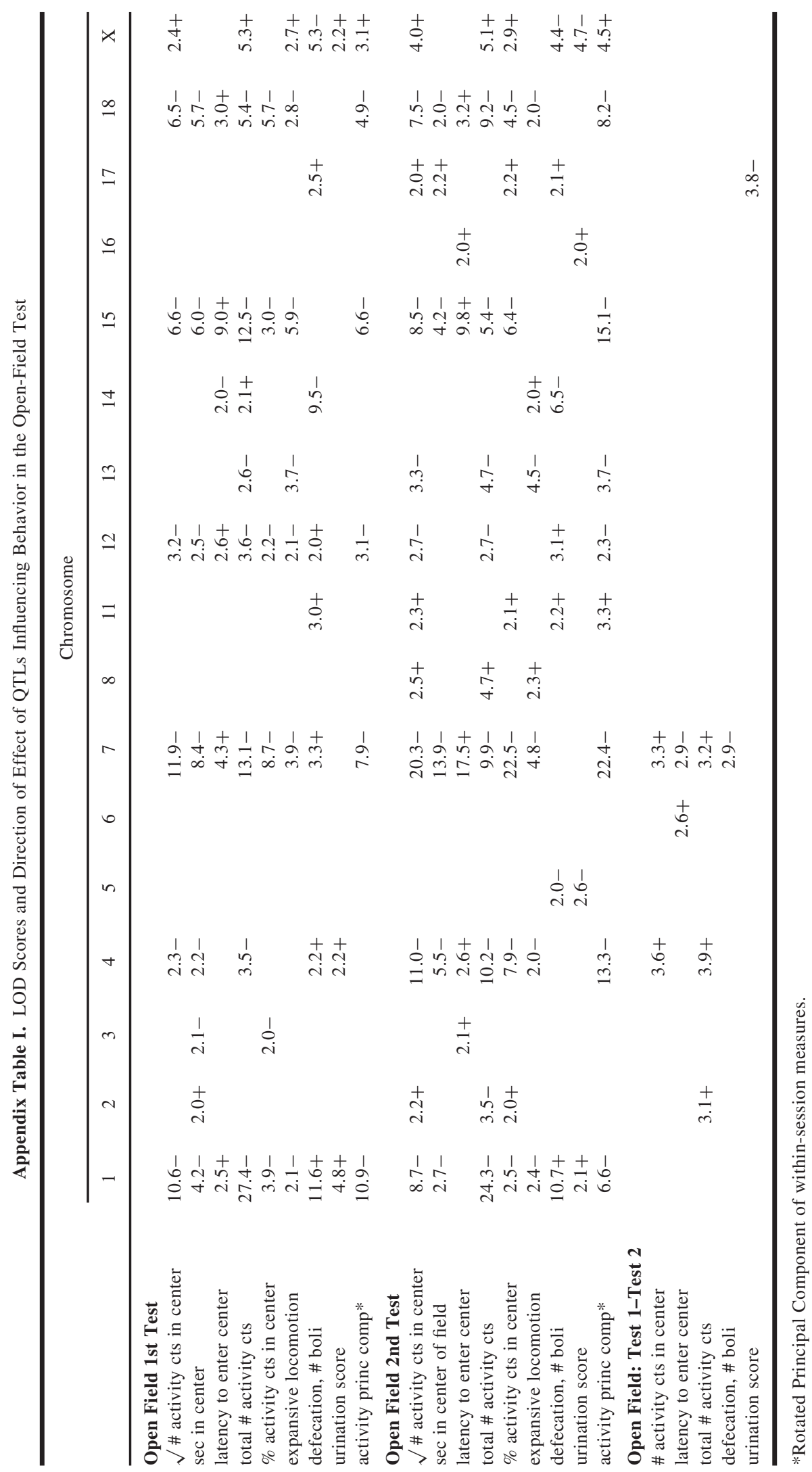




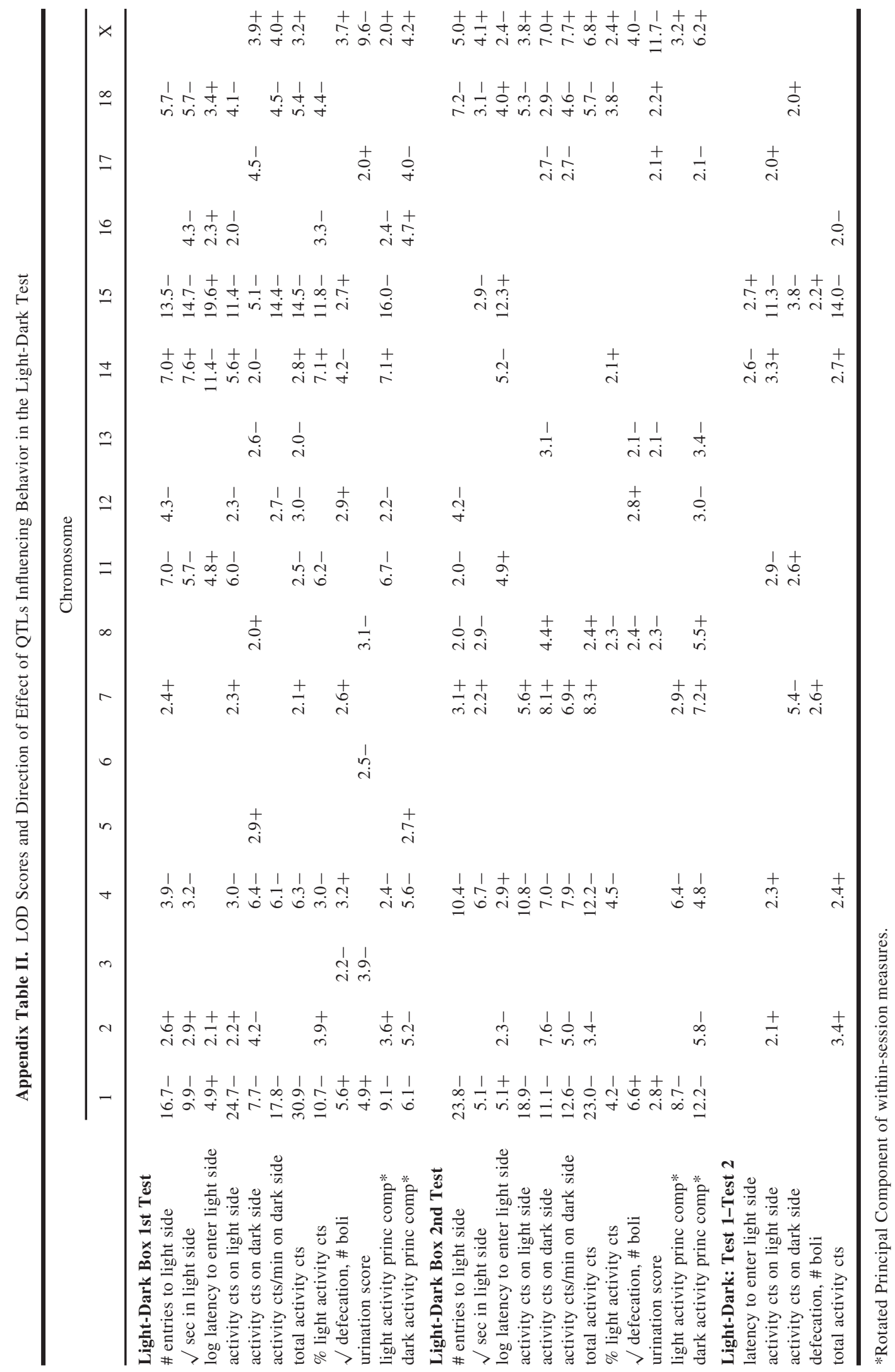




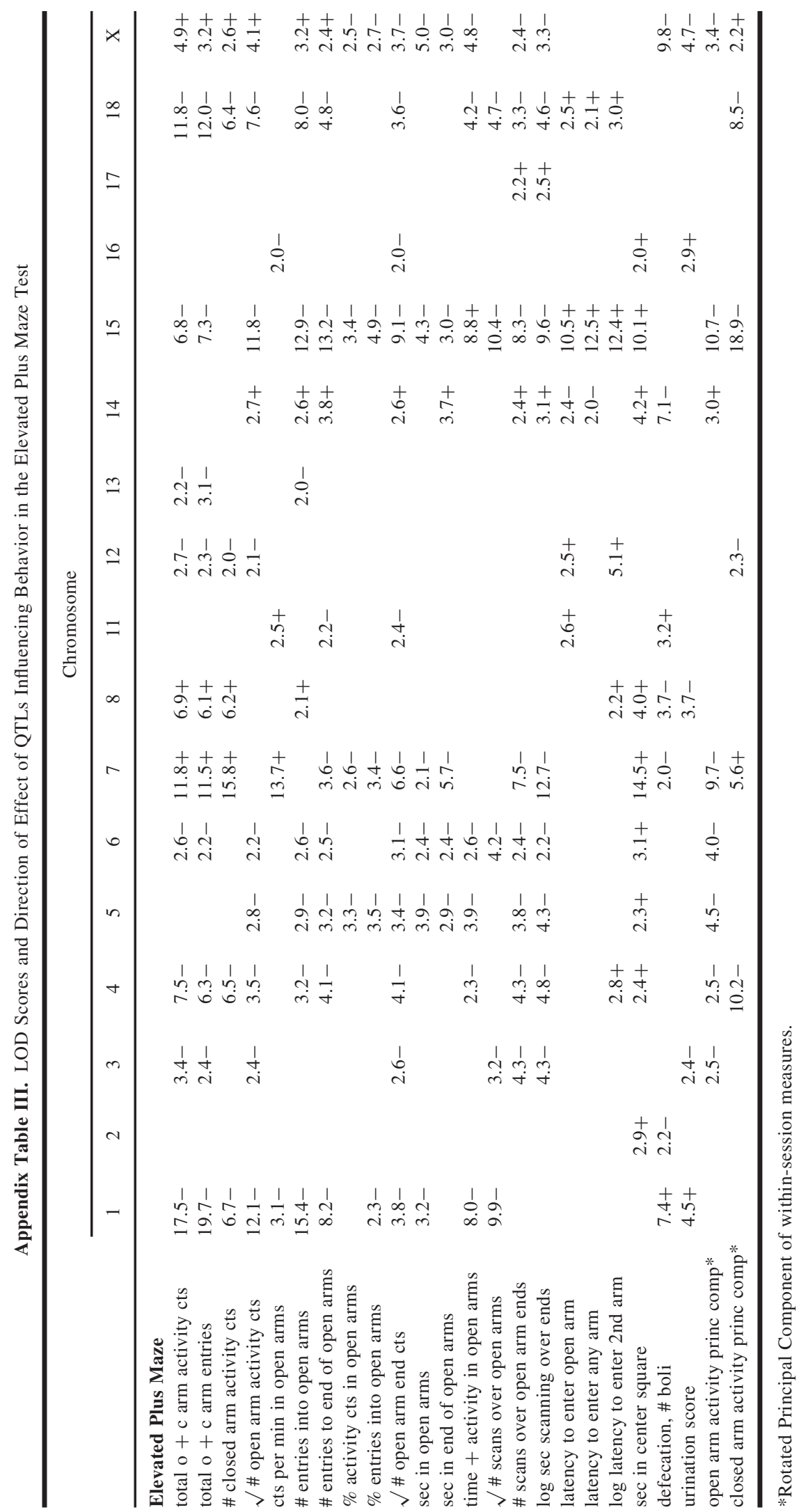



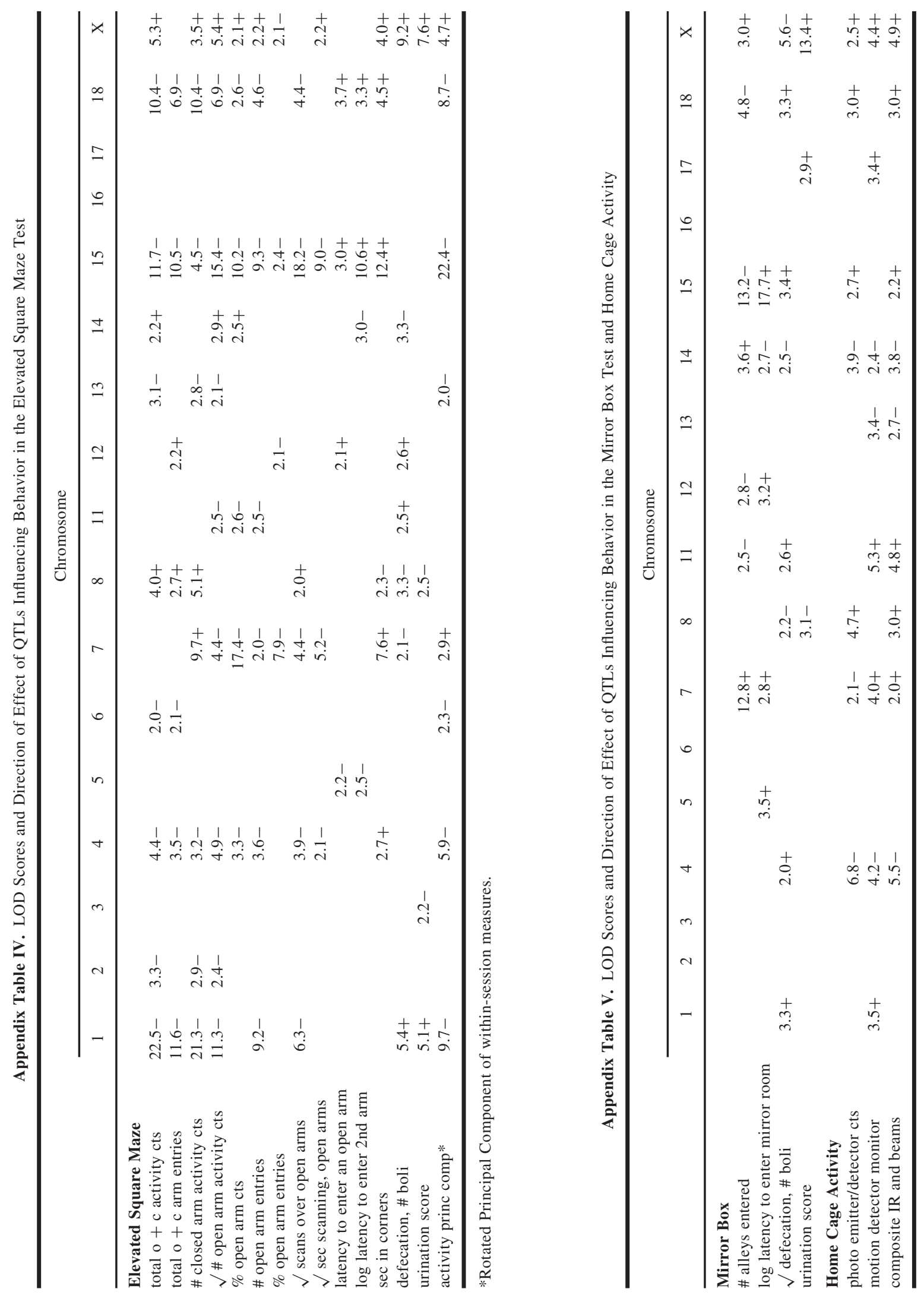


\section{ACKNOWLEDGMENTS}

This work was supported by U.S. National Institute of Mental Health Grant MH53480 to David W. Fulker, Norman D. Henderson, Jonathan Flint, and John C. DeFries. The Wellcome Trust supports Jonathan Flint. Maria Grazia Turri was a Scatcherd European scholar, supported by a fellowship from the Italian Telethon. David Fulker was instrumental in the design of this project but unfortunately did not live to see its completion. Behavioral phenotyping was carried out under the supervision of Norman Henderson and genotyping under the supervision of Jonathan Flint and Maria Turri. Several people were instrumental in the completion of this project. Jerry Salazer supervised animal production. Toby Siffert played a major role in apparatus design and data collection hardware and software. Theresa Tritto supervised extensive pilot studies. Michelle Bohl and Colleen Cykowski supervised behavioral testing, and Susmita Datta assisted in the genotyping of F2 mice. We would also like to acknowledge the support of Allan C. Collins throughout the project and the helpful suggestions provided by Robert Plomin and an anonymous reviewer.

\section{REFERENCES}

Aguilar, R., Gil, L., Flint, J., Gray, J. A., Dawson, G. R., Driscoll, P., Gimenez-Llort, L., Escorihuela, R. M., FernándezTeruel, A., and Tobẽna, A. (2002). Learned fear, emotional reactivity and fear of heights: A factor analytic map from a large $F(2)$ intercross of Roman rat strains. Brain Res. Bull. 57:17-26.

Barlow, D. H. (2000). Unraveling the mysteries of anxiety and its disorders from the perspective of emotion theory. Am. Psychol. 55: $1247-1263$.

Belzung, C. (2001). Rodent models of anxiety-like behaviors: Are they predictive for compounds acting via non-benzodiazepine mechanisms? Curr. Opin. Invest. Drugs 2:1108-1111.

Belzung, C., and Le Pape, G. (1994). Comparison of different behavioral test situations used in psychopharmacology for measurement of anxiety. Physiol. Behav. 56:623-628.

Cattell, R. B. (1966). The meaning and strategic use of factor analysis. In R. B. Cattell (ed.), Handbook of Multivariate Experimental Psychology. Chicago: Rand McNally.

Collins, A. C., Miner, L. L., and Marks, M. J. (1988). Genetic influences on acute responses to nicotine and nicotine tolerance in the mouse. Pharmacol. Biochem. Behav. 30:269-278.

Collins, R. L. (1964). Inheritance of avoidance conditioning in mice: A diallele study. Science 143:1188-1190.

Costall, B., Jones, B. J., Kelly, M. E., Naylor, R. J., and Tomkins, D. M. (1989). Exploration of mice in a black and white test box: Validation as a model of anxiety. Pharmacol. Biochem. Behav. 32:777-785.

Crawley, J. N. (1985). Exploratory behavior models of anxiety in mice. Neurosci. Biobehav. Rev. 9:37-44.

Darvasi, A., and Soller, M. (1997). A simple method to calculate resolving power and confidence interval of QTL map location. Behav. Genet. 27:125-132.
DeFries, J. C. (1969). Pleiotropic effects of albinism on open field behaviour in mice. Nature 221:65-66.

DeFries, J. C., Gervais, M. C., and Thomas, E. A. (1978). Response to 30 generations of selection for open field activity in laboratory mice. Behav. Genet. 8:3-13.

DeFries, J. C., and Hegman, J. P. (1970). Genetic analysis of openfield behavior. In G. Lindzey and D. D. Thiessen (eds.), Contributions to Behavior Genetic Analysis:The Mouse as a Prototype. New York: Appleton-Century-Crofts.

DeFries, J. C., Hegmann, J. P., and Weir, M. W. (1966). Open-field behavior in mice: Evidence for a major gene effect mediated by the visual system. Science 154:1577-1579.

Fernandes, C., González, M. I., Wilson, C. A., and File, S. E. (1999). Factor analysis shows that female rat behavior is characterised primarily by activity, male rats are driven by sex and anxiety. Pharmacol. Biochem. Behav. 64:731-738.

Flaherty, C. F., Greenwood, A., Martin, J., and Leszczuk, M. (1998). Relationship of negative contrast to animal models of fear and anxiety. Anim. Learn. Behav. 26:397-407.

Flint, J. (2003). The analysis of quantitative trait loci that influence animal behaviour. J. Neurobiol. 54:46-77.

Flint, J., Corley, R., DeFries, J. C., Fulker, D. W., Gray, J. A., Miller, S., and Collins, A. C. (1995). A simple genetic basis for a complex psychological trait in laboratory mice. Science 269:1432-1435.

Gershenfeld, H. K., Neumann, P. E., Mathis, C., Crawley, J. N., Li, X., and Paul, S. M. (1997). Mapping quantitative trait loci for open-field behavior in mice. Behav. Genet. 27:201-210.

Gershenfeld, H. K., and Paul, S. M. (1997). Mapping quantitative trait loci for fear-like behaviors in mice. Genomics 46:1-8.

Gray, J. A. (1987). The Psychology of Fear and Stress. Cambridge: Cambridge University Press.

Gray, J. A., and McNaughton, N. (2000). The Neuropsychology of Anxiety. Oxford: OUP.

Hegmann, J. P., and DeFries, J. C. (1968). Open-field behavior in mice: Genetic analysis of repeated measures. Psychon. Sci. 13:27-28.

Henderson, N. (1979). Genetic correlations between brain size and some behaviors of housemice. In M. Hahn, C. Jensen, and B. Dudek (eds.), Development and Evolution of Brain Size. New York: Academic Press.

Henderson, N. D. (1989). Interpreting studies that compare high- and low-selected lines on new characters. Behav. Genet. 19:473-502.

Hogg, S. (1996). A review of the validity and variability of the elevated plus maze as an animal model of anxiety. Pharmacol. Biochem. Behav. 54:21-30.

Knott, S. A., and Haley, C. S. (2000). Multitrait least squares for quantitative trait loci detection. Genet. 156:899-911.

Lander, E., and Kruglyak, L. (1995). Genetic dissection of complex traits: Guidelines for interpreting and reporting linkage results. Nat. Genet. 11:241-247.

Landgraf, R., and Wigger, A. (2002). High vs. low anxiety-related behavior rats: An animal model of extremes in trait anxiety. Behav. Genet. 32:301-314.

Lincoln, S., Daly, M., and Lander, E. (1992). Mapping Genes Controlling Quantitative Traits with MAPMAKER/QTL 1.1. Cambridge, MA: Whitehead Institute Technical Report.

Lord, F. (1980). Applications of Item Response Theory to Practical Testing Problems. Hillsdale, N.J.: Erlbaum.

Nadeau, J. H., and Frankel, W. N. (2000). The roads from phenotypic variation to gene discovery: Mutagenesis versus QTLs. Nat. Genet. 25:381-384.

Pellow, S., Chopin, P., File, S., and Briley, M. (1985). Validation of open: Closed arms entries in an elevated plus maze as a measure of anxiety in the rat. J. Neurosci. Methods 14: $149-167$.

Preacher, K., and MacCallum, R. (2002). Exploratory factor analysis in behavior genetics research: Factor recovery with small sample sizes. Behav. Genet. 32:153-161. 
Ramos, A., and Mormede, P. (1998). Stress and emotionality: A multidimensional and genetic approach. Neurosci. Biobehav. Rev. 22:33-57.

Rodgers, R. J. (1997). Animal models of 'anxiety': Where next? Behav. Pharmacol. 8:477-496.

Rodgers, R. J., and Cole, J. C. (1994). The elevated plus maze: Pharmacology, methodology and ethology. In S. J. Cooper (ed.), Ethology and Psychopharmacology. Chichester: Wiley.

Royce, J. R., Poley, W., and Yeudall, L. T. (1973). Behavior-genetic analysis of mouse emotionality. I. Factor analysis. J. Comp. Physiol. Psychol. 83:36-47.

Shepherd, J. K., Grewal, S. S., Fletcher, A., Bill, D. J., and Dourish, C. T. (1994). Behavioural and pharmacological characterisation of the elevated "zero-maze" as an animal model of anxiety. Psychopharmacology (Berl) 116:56-64.

Stoll, M., Cowley, A. W., Jr., Tonellato, P. J., Greene, A. S., Kaldunski, M. L., Roman, R. J., Dumas, P., Schork, N. J., Wang, Z., and Jacob, H. J. (2001). A genomic-systems biology map for cardiovascular function. Science 294: $1723-1726$.
Toubas, P. L., Abla, K. A., Cao, W., Logan, L. G., and Seale, T. W. (1990). Latency to enter a mirrored chamber: A novel behavioral assay for anxiolytic agents. Pharmacol. Biochem. Behav. 35:121-126.

Trullas, R., and Skolnick, P. (1993). Differences in fear motivated behaviors among inbred mouse strains. Psychopharmacology 111:323-331.

Turri, M. G., Datta, S. R., DeFries, J., Henderson, N. D., and Flint, J. (2001a). QTL analysis identifies multiple behavioral dimensions in ethological tests of anxiety in laboratory mice. Curr. Biol. 11:725-734.

Turri, M. G., Henderson, N. D., DeFries, J. C., and Flint, J. (2001b) Quantitative trait locus mapping in laboratory mice derived from a replicated selection experiment for open-field activity. Genetics 158:1217-1226.

Van Abeelen, J. H., van der Kroon, P. H., and Bekkers, M. F. (1973). Mice selected for rearing behavior: Some physiological variables. Behav. Genet. 3:85-90.

Edited by Jeanne Wehner 
Copyright of Behavior Genetics is the property of Kluwer Academic Publishing and its content may not be copied or emailed to multiple sites or posted to a listserv without the copyright holder's express written permission. However, users may print, download, or email articles for individual use. 